\title{
Sequential learning, predictability, and optimal portfolio returns
}

\author{
Michael Johannes \\ Arthur Korteweg \\ Nicholas Polson *
}

January 10, 2012

\begin{abstract}
This paper finds statistically and economically significant out-of-sample portfolio benefits for an investor who uses models of return predictability when forming optimal portfolios. The key is that investors must incorporate an ensemble of important features into their optimal portfolio problem including time-varying volatility and time-varying expected returns driven by improved predictors such as measures of yield that include shares repurchase and issuance in addition to cash payouts. In addition, investors need to account for estimation risk when forming optimal portfolios. Prior research document a lack of benefits to return predictability, and our results suggest this was largely due to omitting time-varying volatility and estimation risk. We also study the learning problem of investors, documenting the sequential process of learning about parameters, state variables, and models as new data arrives.
\end{abstract}

\footnotetext{
* Johannes is at the Graduate School of Business, Columbia University, mj335@columbia.edu. Korteweg is at the Graduate School of Business, Stanford University, korteweg@stanford.edu. Polson is at the Graduate School of Business, University of Chicago, ngp@chicagogsb.edu. We thank Martijn Cremers, Darrell Duffie, Wayne Ferson, Stefan Nagel, and seminar participants at Columbia, Duke, Rice, the University of North Carolina, USC Marshall, Yale School of Management, the University of Chicago, the 2009 AFA meetings, the 2008 Conference on Modeling and Forecasting Economic and Financial Time Series with State Space models at the Sveriges Riksbank, the 2009 CREATES conference in Skagen, Denmark, the 2009 SOFiE conference in Lausanne, the 2009 CIREQ-CIRANO Financial Econometrics Conference in Montreal, and the 2009 Quantitative Methods in Finance Symposium at UT Austin for helpful comments. We thank Ravi Pillai for excellent computing support. All errors are our own.
} 


\section{Introduction}

Equity return predictability is widely considered a stylized fact: theory indicates expected returns should time-vary and numerous studies find supporting evidence. For example, Lettau and Ludvigson argue "it is now widely accepted that excess returns are predictable by variables such as dividend-price ratios, earnings-price ratios, dividend-earnings ratios, and an assortment of other financial indicators" (2001, p. 842). Evidence for predictable volatility is so strong to be rarely debated, with predictability introduced via short-run persistence and long-run mean-reversion. This predictability should be very important for investors when making portfolio decisions, as investors should 'time' the investment set, increasing allocations when expected returns are high and/or volatility is low.

A surprising recent finding indicates that there is, in fact, little evidence for expected aggregate equity return predictability, and, moreover, there are no out-of-sample benefits to investors from exploiting this predictability when making optimal portfolio decisions. Goyal and Welch (2008, p. 1456) find that "the evidence suggests that most models are unstable or even spurious. Most models are no longer significant even in-sample. ... Our evidence suggests that the models would not have helped such an investor" who is seeking to use the predictability when forming portfolios. Intuitively, the conclusion is that while there maybe be some evidence for predictability, it is so weak to be of no practical use for investors.

This paper revisits this issue, and we find new results reconciling these seemingly contradictory findings. We find strong evidence that investors can use predictability to improve out-of-sample portfolio performance provided investors incorporate a number of sensible features into their optimal portfolio problems. Investors must account for estimation risk when forming portfolios, they must incorporate time-varying volatility, and use improved predictors that measure total net payouts including share issuances and repurchases (Boudoukh et al., 2007). Our results are not inconsistent with Goyal and Welch (2008), as we find no benefits to expected return predictability using the standard approach, which uses regression models with constant volatility and ignores estimation risk.

Intuitively, an ensemble of additional features is needed because each feature provides only a marginal increase in performance. For example, estimation risk is important because there is substantial uncertainty over the nature of the predictability and ignoring it understates predictive return risk (Brennan (1998), Stambaugh (1999) and Barberis (2000)). However, incorporating estimation risk does not, in and of itself, generate statistically significant out-of-sample improvements for the standard predictability model. The same is true for time-varying volatility. One way to interpret our results is that careful modeling requires accounting for all of the first-order important features, such as predictable expected returns, time-varying volatility and parameter uncertainty. Thus, there is no single 'silver bullet' that generates out-of-sample gains.

Our empirical experiment is straightforward. We consider a Bayesian investor who (a) uses models incorporating yield based expected return predictors and stochastic volatility (SV), (b) learns about the models, parameters and state variables sequentially in real time, revising beliefs 
via Bayes' rule as new data arrives, and (c) computes predictive return distributions and maximizes expected utility accounting for all sources of uncertainty. Thus, our investor faces the same learning problems that econometricians face, a problem discussed in Hansen (2007).

To implement the Bayesian portfolio problem, we need to characterize the posterior distribution at each point in time throughout our sample. We use particle filters to tackle this difficult sequential learning problem. Particle filters are a recursive Monte Carlo approach that generate approximate samples from the posterior distribution that we can use to generate draws from the predictive return distributions to compute optimal portfolio holdings. Particle filters are the dominant approach for sequential state or parameter inference across a range of fields.

After solving the learning problem, our investor maximizes expected CRRA utility over terminal wealth for different time horizons, from one month to two years. In the long-horizon problems, our investor rebalances annually. Ideally, one would solve the recursive long-horizon portfolio problem with intermediate learning, but this is infeasible with multiple unknown parameters. ${ }^{1}$ Given these portfolios, we compute out-of-sample portfolio returns, summarizing performance using standard metrics such as Sharpe ratios and certainty equivalent returns (CEs). CEs are a more relevant benchmark than Sharpe ratios given power utility. This procedure generates a time series of realized, fully out-of-sample returns for various models and datasets (cash dividend yields and net payout yields). To evaluate the statistical significance, we simulate returns under various scenarios, e.g., constant means and variances, and evaluate the models with various forms of predictability to see if the Sharpe ratios or CEs are statistically different from those that would be expected from simpler model specifications. ${ }^{2}$

Empirically, our first set of results indicates that the models with constant volatility, while improving the raw out-of-sample portfolio performance of models with predictability, do not generate large enough improvements to be statistically significant. This implies that taking parameter uncertainty and the improved Boudoukh et al. (2007) payout yield predictor into account provides no statistically significant benefits, when relying on constant volatility models. This is consistent with Goyal and Welch (2008), but actually goes one step further and implies that just accounting for parameter uncertainty (i.e. being a Bayesian) does not generate statistically significant improvements. In some cases, timing based on expected return predictability using the traditional cash dividend measure performs worse than using a model with constant means and variances (accounting for parameter uncertainty in both cases). This result is robust for all risk aversion cases and across all investment horizons that we consider.

Our main result is that incorporating an ensemble of factors significantly improves out-of-sample performance. A specification with time-varying expected returns generated by net-payout yields

\footnotetext{
${ }^{1}$ The Bellman equation generated by the fully dynamic problem is high-dimensional. Essentially, each unknown state and parameter has sufficient statistics, and thus the dimensionality of the Bellman equation is roughly equal to twice the number of unknown parameters and states, on the order of 25 dimensions for even the simplest models. Solving this is not feasible with current computing capabilities.

${ }^{2}$ Although our investor is Bayesian, there are no methodological problems evaluating the out-of-sample returns generated by a Bayesian investor using classical statistical techniques. We thank the Associate Editor and a referee for suggesting this experiment.
} 
and SV when used by an investor who accounts for parameter uncertainty generates statistically significant (at the 5\% level) improvements in CEs and Sharpe ratios. This holds for all risk-aversion and investment horizon combinations, where significance is measured either against a model with constant means and variances or against a model with constant means and time-varying volatilities.

The effects are economically large. For example, in a model with constant means and variances, a Bayesian investor with a risk-aversion of four generates an annualized CE yield of $4.77 \%$ and a monthly Sharpe ratio of 0.089 (annualized Sharpe ratio of about 0.31). In the general model using net-payout yield as the predictor and incorporating stochastic volatility, the investor generates a CE yield of $6.85 \%$ and a Sharpe ratio of 0.155 (annualized, 0.54). The $2 \%$ difference in CE yields generates extremely large gains when compounded over a sample of almost 80 years. The Sharpe ratios are more than $70 \%$ higher. The results are even stronger at longer horizons. Together, the results indicate that an ensemble of factors generates statistically and economically significant improvements.

It is also important to note that models with constant expected returns and time-varying volatility do not generate statistically significant returns. Thus, we find no evidence for pure volatility timing, even if the investor accounts for parameter uncertainty. ${ }^{3}$ If the cash dividendyield is used instead of net-payout yields along with time-varying volatility, we find statistically strong improvements, but not as large as the improvements generated by the net payout yield measure. We also consider a drifting coefficients specification, but this model does not generate statistically significant results when compared to a SV model with constant expected returns.

We also report a number of interesting results associated with real-time sequential learning. We find evidence that learning can take a significant amount of time, which should not be surprising given the persistence of volatility and expected returns. This does, however, explain why incorporating estimation risk is important, as there is significant uncertainty over parameter estimates even after observing decades of data. We also discuss differences that can arise from pure statistical model selection-finding the models with the highest posterior probability-and finding the models that perform best in terms of optimal portfolios.

We connect our models to the recent results in Pastor and Stambaugh (2011) on term structures of predictive variances. They find that predictive return volatility does not necessarily fall as the time-horizon increases, in contrast to what would happen with i.i.d. returns and in contrast to popular belief. They document this feature in the context of a 'predictive system', in which the relationship between the predictor variables and expected returns is imperfect. The predictive volatility in a model can increase with horizon due to parameter and state variable uncertainty. We perform the same experiments as Pastor and Stambaugh (2011) in the context of our models, and find similar results. Although our models are not a formal imperfect predictive system, our

\footnotetext{
${ }^{3}$ To our knowledge, there is no published evidence for volatility timing based on aggregate equity returns over long sample periods. Fleming, Kirby, and Ostdiek (1998) consider a multivariate asset problem using data from 1982-1996 and study time-varying second moments, which include correlations. We discuss this work in detail below. Yan (2005) considers a problem with many individual stocks and factor stochastic volatility. Bandi, Russell, and Zhu (2008) consider multiple individual stocks and volatility timing using intraday high-frequency equity returns.
} 
results indicate that the increasing predictive volatility as a function of time-horizon is more general feature, as it appears in models other than those considered in Pastor and Stambaugh (2011).

The rest of the paper is as follows. Section 2 describes the standard approach for evaluating predictability via out-of-sample returns, the models we consider, and our methodology. Section 3 reports our results on sequential inference, including parameter estimates and model probabilities, and the out-of-sample portfolio results, and Section 4 concludes.

\section{Evaluating predictability via out-of-sample portfolio performance}

\subsection{The standard approach}

The standard approach considers a model of the form

$$
r_{t+1}=\alpha+\beta x_{t}+\sigma \varepsilon_{t+1}
$$

where $r_{t+1}$ are monthly log excess returns on the CRSP value-weighted portfolio, $x_{t}$ is a predictor variable, $\varepsilon_{t}$ is a mean-zero constant variance error term, and the coefficients $\alpha, \beta$, and $\sigma^{2}$ are 'fixed but unknown' parameters. The dividend yield is the most common predictor, defined as the natural logarithm of the previous year's cash payouts divided by the current price. Standard full-sample statistical tests for predictability estimate the models on a long historical sample commonly starting in $1927 .{ }^{4}$ It is possible to incorporate multiple predictors, but this paper follows the literature and focuses on univariate regression models.

Although statistical significance is important for testing theories, measures of economic performance, such as the performance of optimal portfolios out-of-sample, are arguably more appropriate and require that investors could identify and take advantage of the predictability in real-time. Typical implementations of out-of-sample portfolio experiments such as Goyal and Welch (2008) use regression models like the one above combined with the assumption of normally distributed errors to form optimal portfolios. An investor finds portfolio weights between aggregate equities and the risk-free rate by maximizing one-period expected utility, assuming a power or constant relative risk aversion utility function, using the predictive distribution of returns induced by the regression model. The initial parameter estimates are estimated based on a training sample, and are re-estimated as new data arrives. Point estimates for the parameters are used to predict future returns. This is called the plug-in method. As mentioned earlier, Goyal and Welch (2008) find no benefits to an investor who follows this procedure using a wide range of predictors. ${ }^{5}$ In particular, they find no benefits for the 'classic' predictor variable, cash dividend yield.

\footnotetext{
${ }^{4}$ For recent results in this area and extensive citations, see Shiller (1981), Hodrick (1992), Stambaugh (1999), Avramov (2002), Cremers (2002), Ferson et al. (2003), Lewellen (2004), Torous, Valkanov, and Yan (2004), Campbell and Yogo (2006), Cochrane (2008), Ang and Bekaert (2007), Xia (2007), Campbell and Thompson (2008), Lettau and Van Nieuwerburgh (2008), Pastor and Stambaugh (2009), and Shanken and Tamayo (2011).

${ }^{5}$ Wachter and Warusawitharnana (2009) consider a Bayesian multi-asset portfolio problem with long term bonds, aggregate equity returns, and the risk-free rate. They find out-of-sample benefits for a highly informative prior, but no benefits for other priors. They provide no evidence that the gains are due to timing expected returns in stocks, and, their optimal portfolios maintain large short positions in long-term bonds, which implies they have a large
} 
Prima facie, there are multiple reasons to suspect that the typical approach might perform poorly out-of-sample. First, the regression model above ignores important, first-order, features of equity returns. Most notably, the constant volatility assumption is in strong contrast to observed data, since equity return volatility time-varies. Ignoring this variation could cause optimal portfolios based solely on time-varying expected returns to perform poorly. Moreover, power utility specifications are sensitive to fat tails in the return distribution, a feature absent in the constant volatility, normally distributed shock regression specification, but present in models with timevarying volatility.

Second, the typical approach ignores the fact that the parameters determining the equity premium, $\alpha$ and $\beta$, are estimated with significant amounts of error. In fact, the whole debate about predictability has received so much attention in part because the predictability evidence, while compelling, is still quite weak. By ignoring estimation risk or parameter uncertainty, the standard implementation understates the total uncertainty, as perceived by an investor. Kandel and Stambaugh (1996) and Barberis (2000) document the important role of parameter uncertainty when forming optimal portfolios.

Third, the linear regression model assumes that the relationship between $x_{t}$ and $r_{t+1}$ is timeinvariant. Theoretically, certain asset pricing models, such as Menzly, Santos and Veronesi (2004) or Santos and Veronesi (2006), imply that the relationship between the equity premium and $x_{t}$ varies over time. Empirically, Paye and Timmerman (2006), Lettau and Van Nieuwerburgh (2008), Dangl and Halling (2011), and Henkel et al. (2011) find evidence for time-variation in the relationship between returns and common predictors.

Fourth, most out-of-sample implementations based on expected return predictability focus on the dividend-yield, which measures payouts of firms via cash dividends. As argued by Boudoukh et al. (2007), an expanded measure of payout that includes share repurchases is a far more effective predictor. In fact, they argue that there is no evidence that cash-dividends is a significant predictor but net payout is strongly significant. For all of these reasons, it may not at all be surprising that the standard approach performs poorly out-of-sample.

The goal of this paper is to introduce extensions to deal with these features and to re-evaluate the out-of-sample performance. The next section introduces the models and our empirical approach.

\subsection{Our approach}

\subsubsection{Models}

We consider a number of extensions to the baseline regression model. The first allows volatility to vary over time,

$$
r_{t+1}=\alpha+\beta x_{t}+\sqrt{V_{t+1}^{r}} \varepsilon_{t+1},
$$

negative bond risk premium. Thus, the gains are likely due to bond and not stock positions. The gains they find are quite modest, relative to the gains we document below. Wachter and Warusawitharnana (2011) consider a related problem with dividend-yield timing, but do not consider out-of-sample returns. 
where $V_{t+1}^{r}$ evolves via a log-volatility specification (Jacquier, Polson and Rossi, 1994, 2005),

$$
\log \left(V_{t+1}^{r}\right)=\alpha_{r}+\beta_{r} \log \left(V_{t}^{r}\right)+\sigma_{r} \eta_{t+1}^{r}
$$

In choosing the log-specification, the goal is to have a parsimonious specification insuring that volatility is stochastic, positive, and mean-reverting. Volatility predictability arises from its persistent but mean-reverting behavior.

Time-varying volatility has direct and indirect effects on optimal portfolios. The direct effect is through the time-variation in the investment set generated by stochastic and mean-reverting volatility, as investors will 'time' volatility, increasing or decreasing equity allocations as volatility changes over time. This effect is ignored in constant volatility regression models. There is also an indirect effect because time-varying volatility implies that the signal-to-noise ratio for learning about expected return predictability varies over time. To see this, note that time- $t$ log-likelihood function for the parameters controlling equity premium, conditional on volatility, is

$$
\ln \left(L\left(\alpha, \beta \mid r_{t+1}, x_{t}, V_{t+1}^{r}\right)\right)=c_{t}-\frac{1}{2} \frac{\left(r_{t+1}-\alpha-\beta x_{t}\right)^{2}}{V_{t+1}^{r}},
$$

where $c_{t}$ is a constant that does not depend on the parameters. In models with constant volatility, $V_{t}^{r}=\sigma^{2}$, the amount of information regarding expected return predictability is constant over time. When volatility time-varies, the information content varies with $V_{t}^{r}$. When $V_{t}^{r}$ is high, there is little information about expected returns, thus, the signal-to-noise to noise ratio is low. Conversely, when $V_{t}^{r}$ is low, the signal-to-noise ratio is high. This is, of course, the usual "GLS vs. OLS" problem that vanishes asymptotically, but can be important in this setting due to small sample issues generated by the high persistence of $x_{t}$ and the relatively low signal-to-noise ratio.

The SV specification has an additional important feature for optimal portfolios: it generates fat-tailed return distributions. The distribution of returns in equation (2) is normally distributed, conditional on $V_{t+1}^{r}$ and the parameters, but the marginal and predictive distribution of returns that integrate out the unobserved volatilities are a scale mixture of normals, which has fat-tails. In addition to fitting the variation in volatility, time-varying volatility is a long-standing explanation for fat tails (see, for example, Rosenberg (1972)). The continuous-time literature has found that SV alone cannot generate enough kurtosis to fit the observed return data at high frequencies, such as daily, but at lower frequencies such as monthly, SV models generate excess kurtosis that is, in fact, consistent with the observed returns. This is discussed in more detail below. We assume the volatility shocks are independent of returns. ${ }^{6}$

\footnotetext{
${ }^{6}$ This significantly simplifies implementation, as the mixture approximation of Kim, Shephard, and Chib (1997) can be used in econometric implementation. The leverage effect is often motivated by negative skewness in equity returns: e.g., at a daily frequency, the skewness of aggregate equity is typically about -2 (see Andersen, Benzoni, and Lund (2002)). The skewness is much less significant at monthly frequencies, roughly -0.49 , and is not statistically different from zero. We estimated a specification incorporating a leverage effect using the full-sample of returns, and the point estimate was only -0.11 , which is much smaller (in absolute value) than typically found at the daily frequency (e.g., Eraker, Johannes, and Polson (2003) and Jacquier, Polson, and Rossi (2004) find values around -0.5).
} 
We also allow the regression coefficient on the predictor variable to vary over time. As mentioned above, some theories imply that this coefficient varies, and there is also empirical evidence suggesting that the loading on predictors such as the dividend-price ratio varies over time (Lettau and Van Nieuwerburgh (2008), Dangl and Halling (2011) and Henkel et al. (2011)). This extension posits that $\beta_{t}$, the regression coefficient, is a mean-reverting process with mean $\beta_{0}$ and auto-covariance $\beta_{\beta}$. The model is

$$
\begin{aligned}
& r_{t+1}=\alpha+\beta_{0} x_{t}+\beta_{t+1} x_{t}+\sqrt{V_{t+1}^{r}} \varepsilon_{t+1}^{r} \\
& \beta_{t+1}=\beta_{\beta} \beta_{t}+\sigma_{\beta} \varepsilon_{t+1}^{\beta},
\end{aligned}
$$

where $\varepsilon_{t+1}^{\beta}$ is i.i.d. normal. It is common to assume that $\beta_{t}$ moves slowly, consistent with values of $\beta_{\beta}$ close to one and $\sigma_{\beta}$ relatively small. Alternatively, a Markov switching process would allow for abrupt changes in the states. The drifting coefficient specification is related to Pastor and Stambaugh (2009), who consider latent specifications of the conditional mean, where the shocks in the conditional mean are correlated with returns and with predictor variables. We discuss the connections in greater detail below.

Based on Stambaugh (1986), we model $x_{t}$ as a persistent but mean-reverting process,

$$
x_{t+1}=\alpha_{x}+\beta_{x} x_{t}+\sqrt{V_{t+1}^{x}} \varepsilon_{t+1}^{x},
$$

where $\beta_{x}<1, \operatorname{corr}\left(\varepsilon_{t}^{r}, \varepsilon_{t}^{x}\right)=\rho$, and $V_{t+1}^{x}$ is the time-varying variance of dividend yields. We assume a standard log-specification for $V_{t+1}^{x}, \log \left(V_{t+1}^{x}\right)=\alpha_{v}+\beta_{v} \log \left(V_{t}^{x}\right)+\sigma_{v} \eta_{t+1}^{v}$, where the errors are standard normal. Incorporating a mean-reverting process for $x_{t}$ is particularly important for optimal portfolios formed over long-horizons, which we consider in addition to monthly horizons. As noted by Stambaugh (1999), mean-reversion in $x_{t}$ generates skewness in the predictive distribution of returns at longer horizons.

We consider the following specifications:

- The 'CV-CM' model has constant means (CM) and constant variances (CV). This is a benchmark model with no predictability.

- The 'CV' model has constant variance, but time-varying expected returns. In equations (2) and (7), this is the special case with $V_{t+1}^{r}=\sigma^{2}$ and $V_{t+1}^{r}=\sigma_{x}^{2}$.

- The 'CV-OLS' is the same model as the CV model, but is implemented using ordinary least squares (OLS) with all data up to time t.

- The 'CV-rolling OLS' is the same model as the CV model, but is implemented using ordinary least squares (OLS) and a rolling window of data.

- The 'CV-DC' is a constant volatility model with drifting regression coefficients.

- The 'SV-CM' model is a SV model with a constant mean, i.e. $\beta=0$, which implies that the equity premium is constant. 
- The 'SV' model is a SV model with time-varying expected returns generated by equation (2).

- The 'SV-DC' model denotes the most general specification with SV and predictability driven by the drifting coefficients model in equations (5) and (6).

All of the models are implemented using a Bayesian approach to account for parameter uncertainty, with the exception of the CV-rolling OLS and CV-OLS implementations, which condition on point estimates. We use these to highlight the impact of parameter uncertainty on out-of-sample performance. We focus on payout yield as a single predictor, but use two measures of 'yield:' the traditional cash dividend yields measure and a more inclusive measures of total payouts via the 'net payout' measure of Boudoukh et al. (2007) which includes share issuances and repurchases.

More general specifications are certainly possible, but our goal is not to find the most general econometric specification. Rather, our goal is to model features of the data that are important for optimal portfolios. These include predictability in expected returns, time-varying volatility, contemporaneous correlation between dividend growth shocks and returns, and drifting coefficients. More general specifications could incorporate non-normal return shocks, leverage effects, additional predictor variables, and a factor stochastic covariance structure for dividend growth and returns. There is a large literature modeling aggregate market volatility developing more involved continuous-time specifications with multiple volatility factors and non-normal jump shocks. These models are typically implemented using daily or even higher frequency data, and it would be very difficult to identify these features with lower frequency monthly data.

Additionally, adding economic restrictions generated by present-value calculations such as those in Koijen and Van Binsbergen (2010) may also improve the model's performance. ${ }^{7}$ These extensions add additional parameters and, more importantly, significantly complicate econometric implementation, making sequential implementation extremely difficult. It is important to note that if our models have any gross misspecification, it should be reflected in poor out-of-sample returns.

\subsubsection{Inference}

We consider a Bayesian investor learning about the unobserved variables, parameters, state variables, and models, sequentially over time. Notationally, let $\left\{\mathcal{M}_{j}\right\}_{j=1}^{M}$ denote the models under consideration. In each model there is a vector of unknown static parameters, $\theta$, and a vector of unobserved state variables $L_{t}=\left(V_{t}^{r}, V_{t}^{x}, \beta_{t}\right)$. The observed data consists of a time series of returns and predictor variables, $y^{t}=\left(y_{1}, \ldots, y_{t}\right)$ where $y_{t}=\left(r_{t}, x_{t}\right)$. The Bayesian solution to the inference problem is $p\left(\theta, L_{t}, \mathcal{M}_{j} \mid y^{t}\right)$, the posterior distribution, for each model specification at each time

\footnotetext{
${ }^{7}$ Koijen and Van Binsbergen's (2010) approach introduces non-linear parameter restrictions related to present values via a Campbell and Shiller (1988) log-linearization, assuming underlying shocks have constant volatility. They expand around stationary means, assuming the conditional variances are constant. This approach is difficult to implement sequentially. Parameters used in the approximations, such as stationary means, are unknown. Additionally, the non-linear parameter constraints significantly complicate Bayesian inference, as the models are no longer conjugate.
} 
point. The marginal distributions $p\left(\theta \mid \mathcal{M}_{j}, y^{t}\right), p\left(L_{t} \mid \mathcal{M}_{j}, y^{t}\right)$ and $p\left(\mathcal{M}_{j} \mid y^{t}\right)$ summarize parameter, state variable, and model inference, respectively.

Out-of-sample experiments require estimation of each models at each time period $t=1, \ldots, T$. This real-time or sequential perspective significantly magnifies any computational difficulties associated with estimating latent variable models. For full-sample inference, Markov Chain Monte Carlo (MCMC) methods are commonly used, but they are too computationally burdensome to use for a sequential implementation. To sample from the posterior distributions, we use a Monte Carlo approach called particle filtering.

Particle filters discretize the support of the posterior distribution, and, as shown by Johannes and Polson (2006) and Carvalho et al. (2010, 2011), work well for parameter and state variable inference in many models with latent states such as log-SV models. Particle filters are fully sequential methods: after summarizing the posterior at time $t$, there is never any need to use the past data as particle filters only use the new data to update previous beliefs. Because of the sequential nature of particle filters, they are computationally much faster than alternatives such as repeated implementation of MCMC methods. This is the main advantage of particle filters, but there is an associated cost: particle filtering methods are not as general or robust as MCMC methods. An online appendix provides an overview of particle filters as well as the details of our filtering algorithm, which is an extension of the methods developed in Johannes and Polson (2006) and Carvalho et al. (2010, 2011).

\subsubsection{Optimal portfolios and out-of-sample performance measurement}

When making decisions, a Bayesian investor computes expected utility using the predictive distribution, which automatically accounts for estimation risk. The posterior distribution quantifies parameter uncertainty or estimation risk. This can be contrasted with frequentist statistics, parameters are 'fixed but unknown' quantities and not random variables, and therefore one cannot define concepts like parameter uncertainty.

Our investor maximizes expected utility over terminal wealth $T$ periods in the future, assuming that the wealth at the beginning of each period is $\$ 1$,

$$
\max _{\{\omega\}} E_{t}\left[U\left(W_{t+T}\right) \mid \mathcal{M}_{j}, y^{t}\right]
$$

where wealth evolves from $t$ to $t+T$ via

$$
W_{t+T}=W_{t} \cdot \prod_{\tau=1}^{T}\left[\left(1-\omega_{t+\tau-1}\right) \exp \left(r_{t+\tau}^{f}\right)+\omega_{t+\tau-1} \exp \left(r_{t+\tau}^{f}+r_{t+\tau}\right)\right]
$$

and $r_{t+\tau}^{f}$ is a zero coupon default-free log bond yield for the period between time $t+\tau-1$ and $t+\tau .^{8}$ The portfolio weight on equities is $\omega_{t+\tau-1}$, and is allowed to vary over the investment horizon. We

\footnotetext{
${ }^{8}$ An earlier version of this paper also considered optimal portfolios generated by model averaging, taking into account the fact that there are multiple models.
} 
consider a range of horizons $T$ from one month $(T=1)$ to two years $(T=24) .{ }^{9}$ In the long horizon problems we allow investors to re-balance their portfolios every year, as in Barberis (2000). We cap the portfolio weights at -2 and +3 . This affects mostly the OLS models (CV-OLS and CV-rolling OLS), whose results look much worse if we leave the weights uncapped. The portfolio weights for the other models are more stable and rarely hit the upper or lower bounds.

We consider a power utility investor,

$$
U\left(W_{t+T}\right)=\frac{\left(W_{t+T}\right)^{1-\gamma}}{1-\gamma}
$$

Expected utility is calculated for each model

$$
E_{t}\left[U\left(W_{t+T}\right) \mid \mathcal{M}_{j}, y^{t}\right]=\int U\left(W_{t+T}\right) p\left(W_{t+T} \mid \mathcal{M}_{j}, y^{t}\right) d W_{t+T}
$$

using equation (9) and the predictive distribution of returns,

$$
p\left(r_{t+\tau} \mid \mathcal{M}_{j}, y^{t}\right)=\int p\left(r_{t+\tau} \mid \theta, L_{t}, \mathcal{M}_{j}, y^{t}\right) p\left(\theta, L_{t} \mid \mathcal{M}_{j}, y^{t}\right) d \theta d L_{t} .
$$

Calculating expected utility in this manner, rational Bayesian investors take all of the relevant uncertainty into account by averaging across the unknown parameters and latent state variables, using the posterior distribution $p\left(\theta, L_{t} \mid \mathcal{M}_{j}, y^{t}\right)$.

Marginalization increases alters the conditional return distribution, increasing variance and generating fat tails. To see this, consider a SV specification where the predictive distribution is

$$
p\left(r_{t+1} \mid \mathcal{M}_{j}, y^{t}\right)=\int p\left(r_{t+1} \mid \theta, V_{t}, \mathcal{M}_{j}, y^{t}\right) p\left(V_{t} \mid \theta, \mathcal{M}_{j}, y^{t}\right) p\left(\theta \mid \mathcal{M}_{j}, y^{t}\right) d \theta d V_{t}
$$

and $p\left(r_{t+1} \mid \theta, V_{t}, \mathcal{M}_{j}, y^{t}\right)$ is the normally distributed conditional return distribution, $p\left(V_{t} \mid \theta, \mathcal{M}_{j}, y^{t}\right)$ is the filtered distribution of the stochastic variance, and $p\left(\theta \mid \mathcal{M}_{j}, y^{t}\right)$ is the parameter posterior distribution at time $t$. Although the return distribution is conditionally normal, the predictive distribution will have higher variance and fat tails generated by marginalizing out the uncertainty in volatility and the other parameters. Thus, although the shocks are normally distributed, predictive return distributions are generally non-normal. This non-normality is minor in constant volatility models, but substantial when volatility time-varies. This is important for fitting fattailed aggregate equity returns. Our power utility specification takes into account the conditional non-normalities, which can be important (see also Brandt et al., 2005, Harvey and Siddique, 2000, and Harvey et al., 2010).

At each time period, our investor finds portfolio weights to maximize expected utility. The investor holds the assets for a given period, realizes gains and losses, updates posterior distributions, and then recomputes optimal portfolio weights. This procedure is repeated for each time period generating a time series of out-of-sample returns. Using this time series, standard summary statistics such as certainty equivalent (CE) yields and Sharpe ratios are computed to summarize

\footnotetext{
${ }^{9}$ Previous versions of the paper considered horizons up to 10 years, with similar results.
} 
portfolio performance. Given that the portfolios were formed by maximizing a power utility specification, CE yields are more appropriate. For some models, we will document a strong disagreement between $\mathrm{CE}$ yields and Sharpe ratios, which are generated by the fact that Sharpe ratios do not take into account tail behavior.

\subsubsection{Evaluating statistical significance}

To assess the statistical significance of the CE yields and Sharpe ratios, we perform extensive Monte Carlo simulations to construct finite sample distributions of the performance statistics. Our base simulations consider a null model with no predictability-constant means and variances- that is calibrated to match the full-sample returns. Then, given returns simulated from this null model, we estimate each of our models sequentially using the same estimation procedures that we used on the real data. We repeat this 500 times for each model specification. ${ }^{10}$ From this, we obtain a distribution of CE yields and Sharpe ratios that we can use to assess if the statistics obtained from the real-world data are statistically significantly different from those generated in the null model.

We also consider the null of a SV model with a constant mean. This provides a benchmark SV specification without time-varying expected returns, allowing us to discriminate between timing based solely on volatility and timing based jointly on expected returns and volatility. This is important because SV, as discussed above, can have both direct and indirect effects on the optimal portfolios, the former through volatility timing and latter via time-varying signal-to-noise ratios. As in the previous case, we simulate returns and then re-estimate models for each of the 500 simulated series using the same procedures used on real data.

\section{Empirical Results}

We use monthly log-excess returns from the value-weighted NYSE-AMEX-NASDAQ index (including distributions) minus the 1-month Treasury bill rate from Ibbotson and Associates over the period $1927-2007$ :

$$
r_{t+1}=\ln \left(\left(P_{t+1}+D_{t+1}\right) / P_{t}\right)-\ln \left(1+r_{t}^{f}\right) .
$$

Here $D_{t+1}$ are the dividends obtained during period $t$, and $P_{t+1}$ is the ex-dividend price. The dividend yield regressor is constructed as the natural logarithm of the sum of the previous twelve months of dividends (from CRSP) divided by the current price, as in Cochrane (2008). The net payout measure is from Boudoukh et al. (2007), which starts in 1927 and ends in 2007. This measure includes both dividends and net equity repurchases (repurchases minus issuances) over the last twelve months, scaled by the current price, and can be obtained from the authors' website.

\footnotetext{
${ }^{10}$ This is extremely computationally intensive. Estimating each of the models with latent variables (drifting coefficients or stochastic volatility models) and forming portfolios takes roughly 1 day on a desktop machine. We run 500 simulations for 8 models for both the dividend-yield and payout-yield data. To perform this experiment, we used a large scale supercomputing cluster, which after efficiently programmed, took almost 6 weeks of cluster computing time.
} 
The choice of monthly time horizon is motivated by the past literature. Since SV movements are often high frequency, monthly data will be more informative than lower frequencies such as annual. In addition, we analyze optimal portfolio allocation problems that have typically been analyzed using data at the monthly frequency, see Kandel and Stambaugh (1996), Stambaugh (1999), or Barberis (2000). Figure 1 provides time series of the regressors, OLS regression estimates, and t-statistics. The panel indicates that net payouts are consistently higher than cash dividends over the sample period but the two are broadly similar. Repurchases used to be quite rare but have increased since the 1980s. Overall, the net payout variable is less persistent than the cash dividend yield because firms deliberately smooth cash dividends (Brav et al., 2005), while the net payout variable contains two additional sources of variation through issuances and repurchases.

Figure 1 provides a time series of the regressors as well as OLS regression coefficient estimates and $t$-statistics for the null hypothesis of $\mathcal{H}_{0}: \beta=0$ sequentially through the sample. The regression estimates and $t$-statistics are cumulative up to time $t$, adding new datapoints as they become available (and keeping all old datapoints). The regression coefficients and the associated $t$-statistics are consistently higher for net payout yield than for cash dividends over the sample period. One source of the increased significance is the higher frequency movements in net payouts. The $t$-statistics change significantly over time, falling significantly in the late 1990s and increasing back to prior levels by about 2003. This is consistent with the findings in Boudoukh et al. (2007).

Our Bayesian investor uses standard conjugate priors described in the online appendix, which are calibrated as follows. First, we train the priors from 1927 - 1929 by regressing excess market returns on a constant and the predictor. This procedure can be viewed as assuming non-informative priors, and then updating using the likelihood function using the training sample, which results in a proper conjugate prior distribution. For the SV parameters, we run AR(1) regressions using squared residuals on lagged squared residuals. The initial volatility states are drawn from the distribution of the regression volatility estimate over the training period. For time-varying coefficient models, the return and payout ratio regressions are insufficient to pin down the priors so we place some structure on the parameters governing the evolution of $\beta_{t}$. The prior on $\beta_{\beta}$ is calibrated to have mean 0.95 , with standard deviation 0.1 implying a high autocorrelation in $\beta_{t}$. The conditional means and variances are equal for all models for the first out-of-sample dates. This training sample approach is commonly used to generate 'objective' priors.

\subsection{Sequential parameter estimates and predictive returns}

\subsubsection{Sequential parameter estimates}

Our approach generates parameter posteriors for each time period, for each model specification

and for both predictors. This section discusses the constant volatility (CV) model estimated using the net payout yield measure, and results for the other models/datasets are given in the internet appendix. Figure 2 displays sequential summaries of the posterior distribution, reporting for each parameter the posterior mean (solid line) and a $(1,99) \%$ posterior probability interval at each point in time (the grey shaded area). The interval limits are not necessarily symmetric around the 

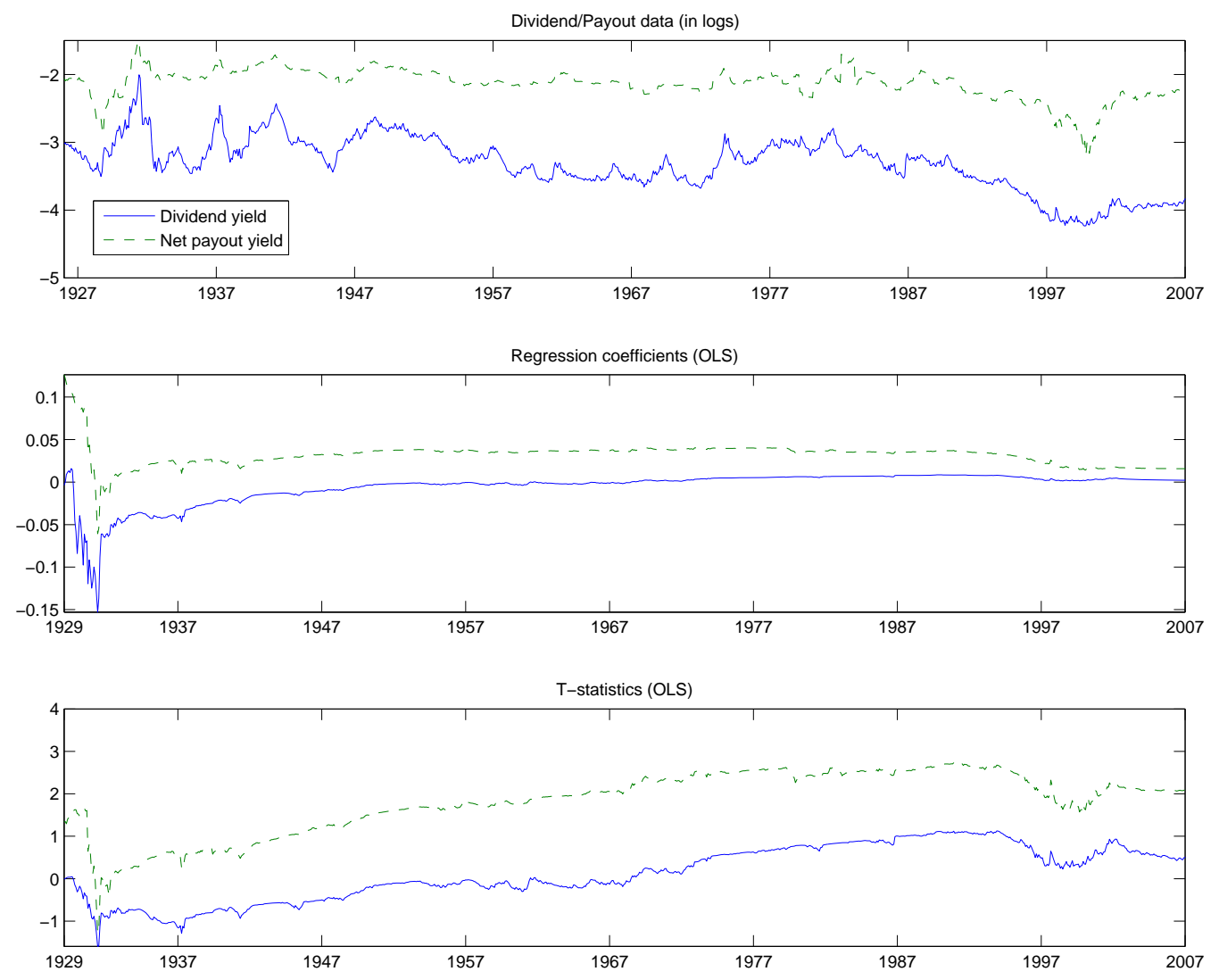

Figure 1: Sequential OLS parameter estimates..

The top panel plots the time-series of the two predictors, dividend (dp) and net payout yield. The middle panel graphs OLS regression coefficients, $\beta$, of the univariate predictability regression:

$$
r_{t}=\alpha+\beta x_{t-1}+\sigma \epsilon_{t},
$$

where $r_{t}$ is the excess market return, the predictor variable $x_{t}$ is either the dividend or net payout yield, and $\epsilon_{t}$ is distributed $\mathcal{N}(0,1)$. We use the entire time series of excess returns, $r_{t}$ up to time $t$ to estimate $\beta$. The bottom panel shows the t-statistics, $t(\beta)$. We use the Amihud-Hurvich (2004) method to adjust for small sample bias. 
mean, because the posteriors are exact finite sample distributions. ${ }^{11}$

There are three notable features in Figure 2. First, the speed of learning varies across parameters. Learning is far slower for expected return parameters, $\alpha$ and $\beta$, and parameters controlling the mean and speed of mean-reversion of the dividend-yield $\left(\alpha_{x}\right.$ and $\left.\beta_{x}\right)$ than for the volatility and correlation parameters. Although standard asymptotics imply a common learning speed, there are differential learning speeds in finite samples. For the expected return parameters, there is still a significant amount of parameter uncertainty even after 30 or 40 years, highlighting how difficult it is to learn expected return parameters due to the low signal-to-noise ratio and the persistence of the yield measure. The slow learning and substantial parameter uncertainty explains why estimation risk might be important for portfolio allocation.

Second, parameter estimates drift over time. This is especially true for the volatility parameters, which occurs because the $\mathrm{CV}$ model has a constant volatility parameter, but is also true for the expected return parameters as estimates of $\alpha$ and $\beta$ slowly decline for the last 20 years of the sample. The estimates of $\beta_{x}$ trend slightly upwards, although the movement is not large. ${ }^{12}$ This 'drifting' of fixed parameter estimates is not necessarily surprising, because the posterior distribution and posterior moments are martingales. Thus, the shocks to quantities such as $E\left(\alpha \mid y^{t}\right)$ are permanent and will not mean-revert.

Third, there is evidence for misspecification. For example, $E\left(\sigma \mid y^{t}\right)$ declines substantially over time, due to omitted SV and the fact that the beginning of the sample has particularly high volatility. Since nearly all studies begin in 1927, discarding this data and starting post-war would create a serious sample selection bias. There are significant shifts in the mean parameters in the net-payout yield equation, $\alpha_{x}$ and $\beta_{x}$, in the late 1970s and early 1980s. Interestingly, Boudoukh et al. (2007) formally test for a structural break and find no evidence, although we use monthly data, whereas they test using annual data. The source of the variation can be found in time series of the regressors in Figure 1, where in the early 1980s the net payout variable has a series of high frequency shocks. As discussed in the web appendix, this is consistent with omitted SV in the dividend yield process. The results from the other models are similar to the CV model, and are discussed in detail in the online web appendix.

One useful way to summarize the differences across models and regressors is to compare the predictability coefficients, i.e. the $\beta$ 's in equation (2). Figure 3 shows that the estimated predictability coefficients differ across models for both datasets. The differences are quite large in the beginning of the sample, especially between the coefficients from constant models and those with SV and time-varying regression coefficients. For the dividend yield data, the SV, SV-DC, and CV parameter estimates are quite similar in the latter part of the sample. For the net-payout

\footnotetext{
${ }^{11}$ Posterior probability intervals (also known as 'credible intervals') represent the probability that a parameter falls within a given region of the parameter space, given the observed data. In Figure 2 the $(1,99) \%$ posterior probability interval represents the compact region of the parameter space for which there is a $1 \%$ probability that the parameter is higher than the region's upper bound, and a $1 \%$ probability that it is lower than the lower bound. Posterior probability intervals should therefore not be interpreted the same way as confidence intervals in classical statistics.

${ }^{12}$ One corroborating piece of evidence is in Brav et al. (2005), who present evidence that the speed of mean reversion for dividends has slowed in the last 50 years, making dividend yields more persistent.
} 

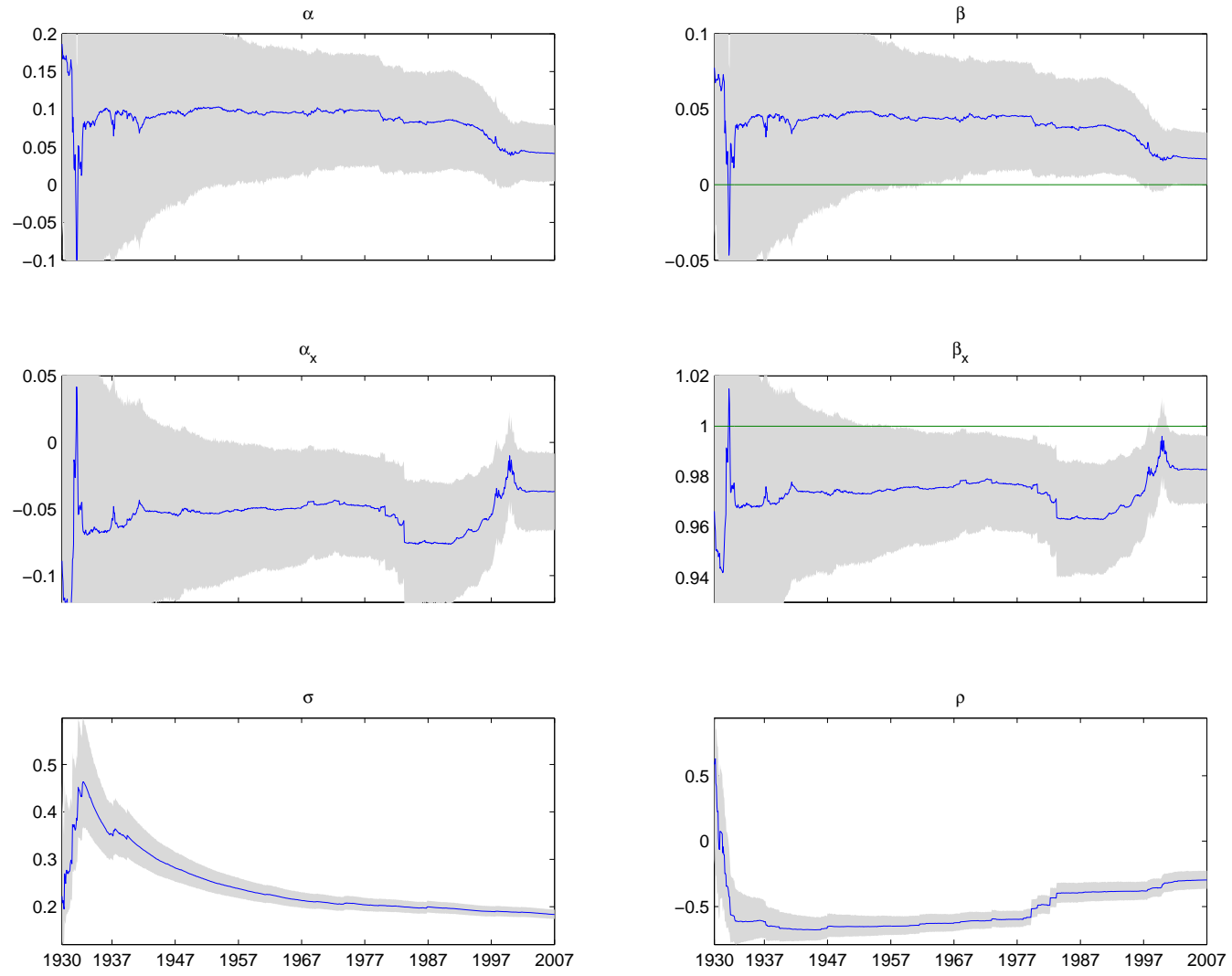

Figure 2: Sequential parameter estimates: CV Model with net payout yield. Sequential parameter estimates for the CV model,

$$
\begin{aligned}
& r_{t+1}=\alpha+\beta x_{t}+\sigma \varepsilon_{t+1}^{r} \\
& x_{t+1}=\alpha_{x}+\beta_{x} x_{t}+\sigma_{x} \varepsilon_{t+1}^{x},
\end{aligned}
$$

where $r_{t+1}$ is the return on the market portfolio in excess of the risk-free rate from month $t$ to month $t+1$. The predictor variable, $x_{t}$, is the net payout yield of Boudoukh et al. (2007). The shocks $\varepsilon_{t+1}^{r}$ and $\varepsilon_{t+1}^{x}$ are distributed standard Normal with correlation coefficient $\rho$. Each panel displays the posterior means and $(1,99) \%$ posterior probability intervals (the grey shaded area) for each time period. Excess market return volatility, $\sigma$, is annualized. 
yield data, there are relatively large differences between the estimates over the entire sample period. The SV models have consistently lower coefficients than the constant volatility models, with the SV coefficients almost half the size of the CV coefficients at points in the 1980s and 1990s. These differences are consistent with a time-varying signal-to-noise ratio. Overall, the models will have varying degrees of statistical evidence in favor of return predictability. The online appendix provides formal Bayesian hypothesis tests of predictability.

\subsubsection{Predictive returns}

Equation (13) provides the one-month ahead predictive return distribution of the excess market return. For each period, we can sequentially compute measures of fat tails, such as the predictive kurtosis. To get a sense of the magnitudes, monthly excess returns have excess kurtosis of about 7.5 over the whole sample. In contrast, the predictive distribution of the baseline CV model with constant volatility has an average (through the sample) excess kurtosis of 0.02 , starting at about 0.15 and declining to less than 0.01 at the end of the sample. This slight excess kurtosis and its decline are due solely to parameter uncertainty, since there is no time-varying volatility in the CV model. Clearly the constant volatility models are unable to fit the tails of the return distribution.

For the SV model, the average predictive excess kurtosis is 8.75, starting around 15 in the beginning of the sample and declining to about 6 at the end of the sample. Thus, our SV model generates kurtosis consistent with the observed data, if marginally higher. The initial higher kurtosis is due to the interaction between parameter uncertainty and SV, as parameter uncertainty in the volatility equation fattens the tails of the volatility distribution, which, in turn, fattens the tails of predictive returns. This is consistent with previous research showing that SV models generate significant kurtosis in the monthly frequency (see Das and Sundaram (1999)). As mentioned earlier, the skewness of returns is modest and not statistically significant at monthly horizons.

Turning to the predictive volatility, a provocative recent paper by Pastor and Stambaugh (2011) shows that predictive return volatility does not necessarily fall as the time-horizon increases, in contrast to popular belief. Denoting $r_{t, t+k}$ as the return from time $t$ to $t+k$, they find that $\operatorname{var}\left(r_{t, t+k} \mid y^{t}\right)$ may increase as a function of $k$, due to parameter and state variable uncertainty. They document this feature in the context of a "predictive system," in which the relationship between the predictor variables and expected returns is imperfect but the conditional volatility of returns is constant.

We perform the same experiments as Pastor and Stambaugh (2011) for our model specifications, which are not formal imperfect predictive systems. The results are in Figure 4. The results indicate that a number of our models generate increasing predictive volatility. Those with drifting coefficients are most similar to those in Pastor and Stambaugh and have a striking increase in the predictive volatility as horizon increases. This is true of both CV and SV specifications with drifting coefficients. The SV model, when volatility is at its long-run mean, generates a slight upward slope in the predictive variance with parameter uncertainty, but a slight decrease conditional on fixed parameters. These results indicate that the increasing predictive volatility as a function of time- 

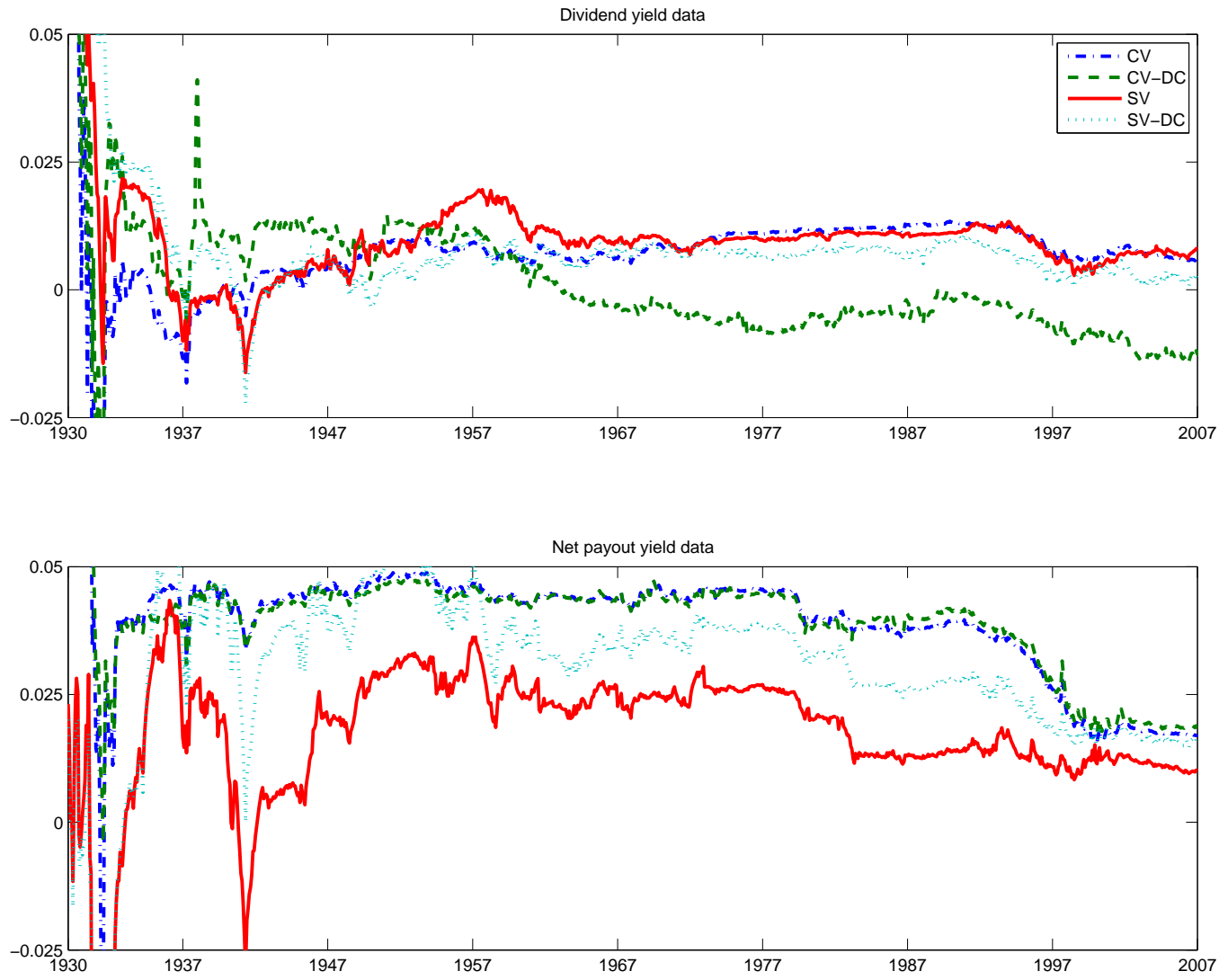

\section{Figure 3: Predictability coefficient}

Time-series plots of the posterior mean of the predictability coefficient, $\beta$, across models and predictor variables. The top panel shows the coefficients of the four models using dividend yield as the predictor variable, and the bottom panel uses net payout yield as the predictor. CV and SV represent models with expected return predictability and constant volatility (CV) and stochastic volatility (SV), respectively. DC stands for drifting coefficients and represents models where the predictability coefficient is allowed to vary over time. 
horizon is a far more general phenomenon, as it appears in models other than those considered in Pastor and Stambaugh (2011).

\subsubsection{Model comparison}

Figure 5 reports posterior model probabilities, $p\left(\mathcal{M}_{j} \mid y^{t}\right)$, throughout the sample. This metric provides a relative comparison of how the models under consideration perform in a predictive sense. The model probabilities accumulate predictive likelihood functions, $p\left(y_{t+1} \mid \mathcal{M}_{j}, y^{t}\right)$, and quantify how well an observation $y_{t+1}$ conforms to its predictive distribution. Thus, the model fit comparisons are fully out-of-sample. The details of these posterior probabilities are given in the internet appendix.

There are three noticeable features. First, there are somewhat rapid revisions in beliefs about models, where the probabilities shift from near one to near zero, or vice versa, over the course of a few months or years. Mechanically, this occurs because observations that are ex-ante unlikely under one model are far more likely under another model. For example, consider the case of cashdividends. The first major shift was in the the 1940s. In this period, volatility dramatically fell and within a year or so the model probabilities for the constant volatility relative to the SV models fell dramatically, which makes sense because the SV model allows volatility can fall rapidly whereas the constant volatility models assume it is a fixed parameter that is learned over time. The subsequent changes are between the drifting coefficients SV model and the standard SV model, which implies the differences were largely first moment related. The next shift was in the late 1960s to mid 1970s, and this shift took more than 5 years to occur. These relatively rapid shifts are novel, as most theoretical work assumes models differences are driven by very slow moving factors.

Second, for both datasets the results eventually and strongly favor models with SV. This is not a surprise, since time-varying volatility is such a strong feature of equity returns. Models with constant volatility have two problems: they can't capture the time-variation in volatility and they have very thin tails, as mentioned above. Finally, different data sources favor different models. The cash dividend data suggests a SV model with constant dividend predictability, while net payout yield suggests a model with SV and drifting coefficients. This is intuitively sensible, since the better signal-to-noise ratio for expected returns when using net-payout yield implies that it is possible to estimate the drifting coefficient, and its underlying parameters, with sufficient accuracy to improve predictive performance. Below, we compare these results with the out-of-sample portfolio results, an alternative predictive measure of model performance.

\subsection{Portfolio Results}

Tables 1 (cash dividends) and 2 (net payout yield) summarize the CEs and Sharpe ratios for the out-of-sample portfolio returns for each model, dataset, risk aversion, and investment horizon. Table 3 reports the mean, standard deviation, skewness, and excess kurtosis statistic for the outof-sample returns for each time horizon and for the case of risk aversion $\gamma=4$. As mentioned earlier, we consider two null models to benchmark significance, a model with constant means and 

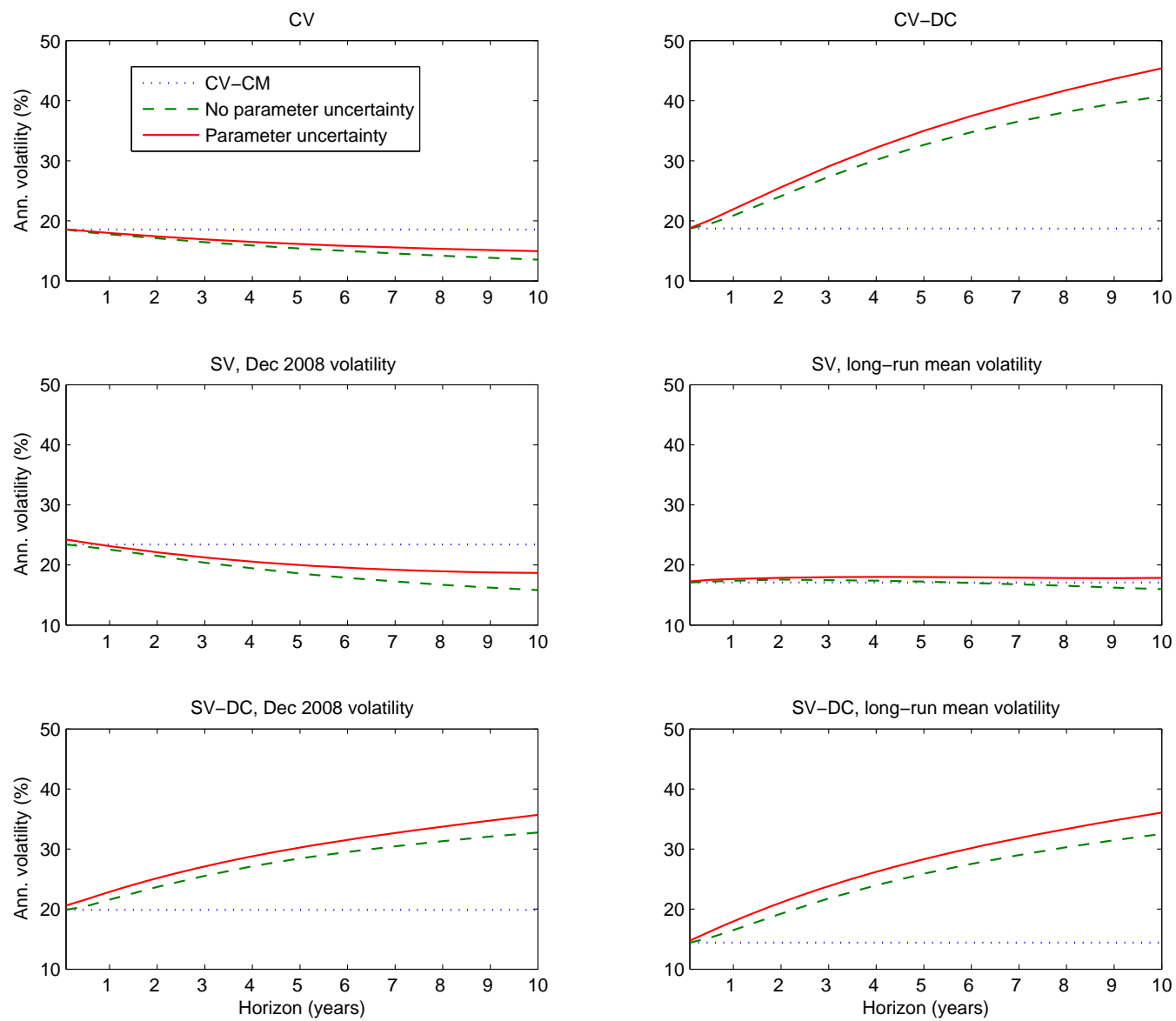

Figure 4: Predictive volatility

This graph shows the term structure of annualized predictive excess return volatilities for various models, using the dividend yield as the predictor variable. Each panel represents a different model. The top left plot shows the predictive volatility for the CV model, which has predictability in expected returns, and constant variance. The striped line ignores parameter uncertainty, whereas the solid line includes the effect of parameter and state uncertainty. As a benchmark, the dotted, horizontal line marks the volatility in the CV-CM model, which has no predictability in either expected returns or variances. The top right plot shows the CV-DC model, with a drifting predictability coefficient and constant variance. The middle row of plots show the stochastic volatility (SV) model, which has both expected return and volatility predictability, at the realized volatility state at the last observation in our dataset, (December 2008, left plot), and at the average volatility level (right plot). Similarly, the bottom row shows the SV-DC model with stochastic volatility and a time-varying expected return predictability coefficient, both in December 2008 (left plot) and at the average level of volatility (right plot). 

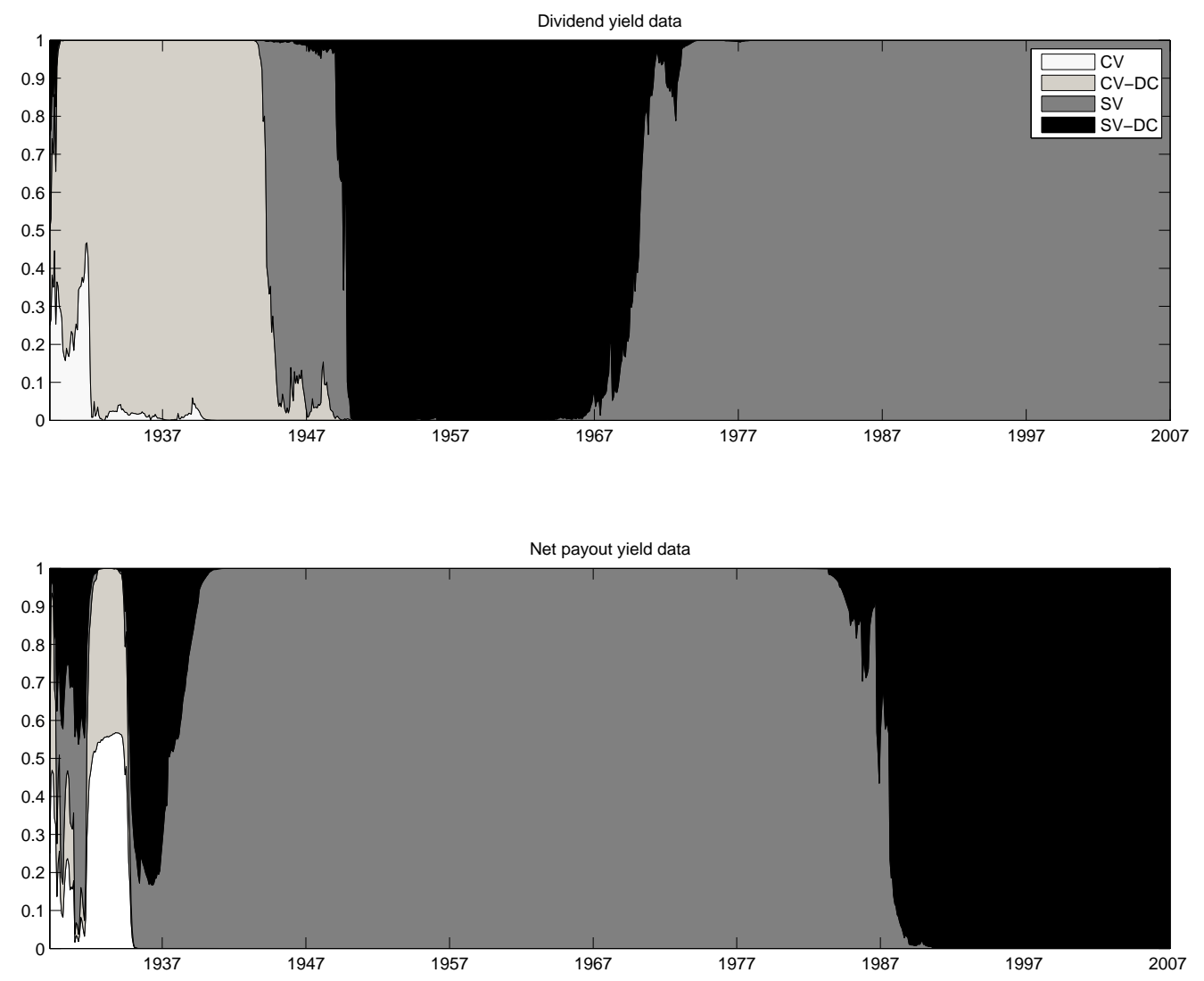

\section{Figure 5: Posterior model probabilities}

This graph shows the time series of posterior model probabilities for four model specifications. The top panel shows the model probabilities using the traditional dividend yield measure as the predictor variable. The bottom panel shows the probabilities using the net payout measure as the predictor. Model probabilities represent the weight of evidence in favor of each given model, relative to the other models, based on the data available at the time. CV and SV represent models with expected return predictability and constant volatility (CV) and stochastic volatility (SV), respectively. DC stands for drifting coefficients and represents models where the predictability coefficient is allowed to vary over time. 
variances and a model with constant means and stochastic volatility.

\subsubsection{Models using dividend yields}

The first results in Table 1 indicate that none of the constant volatility models provide statistically significant improvements. Amongst these poor performing models, the 'best' model is actually the CV-CM model, a model with constant means and variances accounting for parameter uncertainty. This model delivers CE yields of around $4.75 \%$ to $5 \%$ for $\gamma=4 .^{13}$ Interestingly, the models incorporating cash dividend yields as a regressor perform noticeable worse than the constant/mean and volatility model. For example, at the one-month horizon, the CV model (constant volatility with cash-dividend yield predictability) generates a CE yield of $-7 \%$. The two models ignoring parameter uncertainty, the CV-OLS and CV-rolling OLS perform particularly poorly, which shows that accounting for parameter uncertainty improves performance, especially at short horizons. There is a modest improvement for some of the longer horizon cases, but again the results are not statistically significant.

These results are fully consistent with the results in Goyal and Welch (2008). In fact, the results are even stronger as the $\mathrm{CV}$ model accounts for parameter uncertainty. In every case, outof-sample returns incorporating expected return predictability result in worse performance than a constant mean and volatility specification. Even if you are Bayesian and account for parameter uncertainty, there is no consistent statistical or economic evidence for out-of-sample gains for models with constant volatility. The results in panel A of Table 3 provide an alternative view of the poor performance, showing that constant volatility models generate extreme negative skewness and large excess kurtosis. Intuitively, this occurs because the optimal portfolios in these models do not admit the possibility of fluctuating volatility or non-normal returns, and thus the out-ofsample portfolio returns are highly non-normal. The CV-rolling OLS specification has the least non-normalities, but this is largely a denominator effect in the skewness and excess kurtosis as the monthly volatility is over 100 percent.

Turning to the SV specifications, the SV-CM model incorporating time-varying volatility and a constant mean does not provide consistent statistically significant performance improvement, but the CEs and Sharpe ratios do increase noticeably. The effect of stochastic volatility is most clear from the portfolio return statistics in Table 3. Compared to the CV-CM model, the SV-CM model has noticeable better skewness ( -0.56 compared to -1.3$)$ and excess kurtosis $(2.84$ compared to 9.40) for the one-month horizon. The longer horizon portfolio statistics also improve, though not by as much. These results show that SV improves performance along all dimensions: higher Sharpe ratios, less skewness, and lower kurtosis. Despite the improvements generated by the addition of time-varying volatility, the portfolio returns are still not statistically significant from either of the null models. This is an important finding because it indicates that there is no statistical evidence

\footnotetext{
${ }^{13}$ Note that the long-horizon CE yields in the constant means models are different from the 1-month horizon. The reason is that the monthly portfolio weights update each month whereas the long-horizon portfolio weights update annually.
} 
Panel A: Certainty Equivalent returns (in \% per annum)

\begin{tabular}{|c|c|c|c|c|c|c|}
\hline & \multicolumn{3}{|c|}{$\gamma=4$} & \multicolumn{3}{|c|}{$\gamma=6$} \\
\hline & $1 \mathrm{~m}$ & $1 \mathrm{y}$ & $2 \mathrm{y}$ & $1 \mathrm{~m}$ & $1 \mathrm{y}$ & $2 y$ \\
\hline \multicolumn{7}{|c|}{ Constant Volatility models } \\
\hline $\mathrm{CV}-\mathrm{CM}$ & 4.77 & 5.05 & 5.05 & 4.38 & 4.61 & 4.61 \\
\hline CV-OLS & -8.60 & 1.14 & -0.17 & -25.22 & 1.70 & -1.16 \\
\hline CV-rolling OLS & -19.68 & -11.48 & -12.59 & -46.01 & -24.71 & -30.25 \\
\hline $\mathrm{CV}$ & -7.04 & 4.42 & 4.47 & -2.47 & 4.09 & 4.03 \\
\hline CV-DC & -7.64 & 4.04 & 3.95 & -3.29 & 3.72 & 3.51 \\
\hline \multicolumn{7}{|c|}{ Stochastic Volatility models } \\
\hline SV-CM & 5.52 & 5.92 & 5.94 & 4.89 & 5.14 & 5.14 \\
\hline SV & $6.43^{* *, \dagger \dagger}$ & $6.51^{* *, \dagger \dagger}$ & $6.53^{* *, \dagger \dagger}$ & $5.52^{* *, \dagger \dagger}$ & $5.64^{* *, \dagger \dagger}$ & $5.64^{* *, \dagger \dagger}$ \\
\hline SV-DC & $6.56^{* *, \dagger \dagger}$ & 5.85 & 5.68 & $5.64^{* *, \dagger \dagger}$ & 5.17 & 5.02 \\
\hline \multicolumn{7}{|c|}{ Panel B: Sharpe ratios (monthly) } \\
\hline & \multicolumn{3}{|c|}{$\gamma=4$} & \multicolumn{3}{|c|}{$\gamma=6$} \\
\hline & $1 \mathrm{~m}$ & $1 \mathrm{y}$ & $2 y$ & $1 \mathrm{~m}$ & $1 \mathrm{y}$ & $2 y$ \\
\hline \multicolumn{7}{|c|}{ Constant Volatility models } \\
\hline $\mathrm{CV}-\mathrm{CM}$ & 0.089 & 0.099 & 0.099 & 0.088 & 0.101 & 0.101 \\
\hline CV-OLS & 0.005 & 0.019 & 0.015 & -0.001 & 0.016 & 0.006 \\
\hline CV-rolling OLS & 0.102 & 0.108 & 0.110 & 0.100 & 0.108 & 0.109 \\
\hline $\mathrm{CV}$ & 0.025 & 0.074 & 0.076 & 0.023 & 0.069 & 0.067 \\
\hline $\mathrm{CV}-\mathrm{DC}$ & 0.037 & 0.056 & 0.052 & 0.035 & 0.048 & 0.037 \\
\hline \multicolumn{7}{|c|}{ Stochastic Volatility models } \\
\hline $\mathrm{SV}-\mathrm{CM}$ & $0.132^{*, \dagger}$ & 0.125 & 0.125 & $0.133^{*, \dagger}$ & 0.124 & 0.124 \\
\hline SV & $0.143^{* *, \dagger \dagger}$ & $0.144^{* *, \dagger \dagger}$ & $\dagger 0.143^{* *, \dagger \dagger}$ & $0.143^{* *, \dagger \dagger}$ & $0.146^{* *, \dagger \dagger}$ & $\dagger 0.145^{* *}$ \\
\hline SV-DC & $0.143^{* *, \dagger \dagger}$ & $0.135^{*}$ & $0.135^{*}$ & $0.144^{* *, \dagger \dagger}$ & $0.138^{*, \dagger}$ & $0.136^{*}$ \\
\hline
\end{tabular}

Table 1: Portfolio Returns: Dividend Yield Data.

This table shows out-of-sample portfolio returns using the dividend yield as a predictor, for one month, one year and two year investment horizons. Investors have power utility with risk aversion parameter $\gamma$, and allocate their wealth between the market portfolio of stocks and a risk-free oneperiod bond. The certainty equivalent returns in Panel A represent the annualized risk-free return that gives the investor the same utility as the risky portfolio strategy. Panel B shows monthly Sharpe Ratios. CM stands for a model with constant mean (i.e. no predictability), and CV and SV stand for constant and stochastic volatility, respectively. Hence, CV-CM represents a model with constant mean and constant volatility of returns. DC means drifting coefficients and represents models where the coefficient on net payout is allowed to vary over time. CV-OLS uses the OLS point estimates of equation (1), with data up to time $t$. CV-rolling OLS uses a 10-year rolling regression model to form portfolios. * and ${ }^{* *}$ indicate that the result is significant at the $10 \%$ and $5 \%$ level, respectively, based on 500 simulated datasets with constant mean and volatility. $\dagger$ and $\dagger \dagger$ indicate that the result is significant at the $10 \%$ and $5 \%$ level, respectively, based on 500 simulated datasets with constant mean and stochastic volatility. 
Panel A: Certainty Equivalent returns (in \% per annum)

\begin{tabular}{|c|c|c|c|c|c|c|}
\hline & \multicolumn{3}{|c|}{$\gamma=4$} & \multicolumn{3}{|c|}{$\gamma=6$} \\
\hline & $1 \mathrm{~m}$ & $1 \mathrm{y}$ & $2 y$ & $1 \mathrm{~m}$ & $1 \mathrm{y}$ & $2 y$ \\
\hline \multicolumn{7}{|c|}{ Constant Volatility models } \\
\hline CV-OLS & 1.44 & 6.10 & 6.21 & 2.34 & 5.27 & 5.35 \\
\hline CV-rolling OLS & -14.85 & -11.32 & -11.23 & -63.30 & -61.95 & -61.98 \\
\hline $\mathrm{CV}$ & 3.03 & 6.07 & 6.19 & 3.29 & 5.25 & 5.33 \\
\hline CV-DC & 0.77 & 6.19 & 6.27 & 2.00 & 5.33 & 5.40 \\
\hline \multicolumn{7}{|c|}{ Stochastic Volatility models } \\
\hline SV & $6.85^{* *, \dagger \dagger}$ & $7.50^{* *, \dagger \dagger}$ & $7.67^{* *, \dagger \dagger}$ & $5.71^{* *, \dagger \dagger}$ & $6.29^{* *, \dagger \dagger}$ & $6.42^{* *, \dagger \dagger}$ \\
\hline SV-DC & $6.23^{* *, \dagger}$ & $7.22^{* *, \dagger \dagger}$ & $7.36^{* *, \dagger \dagger}$ & $5.36^{* *, \dagger}$ & $6.08^{* *, \dagger \dagger}$ & $6.22^{* *, \dagger \dagger}$ \\
\hline \multirow{2}{*}{\multicolumn{4}{|c|}{ Panel B: Sharpe ratios (monthly) }} & . & . & . \\
\hline & & & & . & . & . \\
\hline & \multicolumn{3}{|c|}{$\gamma=4$} & \multicolumn{3}{|c|}{$\gamma=6$} \\
\hline & $1 \mathrm{~m}$ & $1 \mathrm{y}$ & $2 y$ & $1 \mathrm{~m}$ & $1 \mathrm{y}$ & $2 y$ \\
\hline \multicolumn{7}{|c|}{ Constant Volatility models } \\
\hline CV-OLS & 0.011 & $0.150^{* *, \dagger \dagger}$ & $\dagger 0.151^{* *, \dagger \dagger}$ & 0.011 & $0.149^{* *, \dagger \dagger}$ & $\dagger 0.151^{* *, \dagger}$ \\
\hline CV-rolling OLS & 0.118 & $0.143^{* *, \dagger \dagger}$ & $\dagger 0.146^{* *, \dagger \dagger}$ & 0.113 & $0.132^{*, \dagger}$ & $0.136^{* *, \dagger}$ \\
\hline $\mathrm{CV}$ & 0.045 & $0.155^{* *, \dagger \dagger}$ & $\dagger 0.156^{* *, \dagger \dagger}$ & 0.045 & $0.154^{* *, \dagger \dagger}$ & $\dagger 0.155^{* *, \dagger}$ \\
\hline CV-DC & 0.044 & $0.156^{* *, \dagger \dagger}$ & $\dagger 0.156^{* *, \dagger \dagger}$ & 0.042 & $0.155^{* *, \dagger \dagger}$ & $\dagger 0.156^{* *, \dagger}$ \\
\hline \multicolumn{7}{|c|}{ Stochastic Volatility models } \\
\hline SV & $0.155^{* *, \dagger \dagger}$ & $0.172^{* *, \dagger \dagger}$ & $\dagger 0.172^{* * \dagger \dagger}$ & $0.154^{* *, \dagger \dagger}$ & $0.172^{* *, \dagger \dagger}$ & $\dagger 0.171^{* *, \dagger}$ \\
\hline SV-DC & $0.144^{* *, \dagger \dagger}$ & $0.166^{* *, \dagger \dagger}$ & $\dagger 0.167^{* *, \dagger \dagger}$ & $0.145^{* *, \dagger \dagger}$ & † $0.166^{* *, \dagger \dagger}$ & $\dagger 0.166^{* *, \dagger \dagger}$ \\
\hline
\end{tabular}

Table 2: Portfolio Returns: Net Payout Yield Data.

This table shows out-of-sample portfolio returns using the dividend yield as a predictor, for one month, one year and two year investment horizons. Investors have power utility with risk aversion parameter $\gamma$, and allocate their wealth between the market portfolio of stocks and a risk-free oneperiod bond. The certainty equivalent returns in Panel A represent the annualized risk-free return that gives the investor the same utility as the risky portfolio strategy. Panel B shows monthly Sharpe Ratios. CM stands for a model with constant mean (i.e. no predictability), and CV and SV stand for constant and stochastic volatility, respectively. Hence, CV-CM represents a model with constant mean and constant volatility of returns. DC means drifting coefficients and represents models where the coefficient on net payout is allowed to vary over time. CV-OLS uses the OLS point estimates of equation (1), with data up to time $t$. CV-rolling OLS uses a 10-year rolling regression model to form portfolios. ${ }^{*}$ and ${ }^{* *}$ indicate that the result is significant at the $10 \%$ and $5 \%$ level, respectively, based on 500 simulated datasets with constant mean and volatility. $\dagger$ and $\dagger \dagger$ indicate that the result is significant at the $10 \%$ and $5 \%$ level, respectively, based on 500 simulated datasets with constant mean and stochastic volatility. 

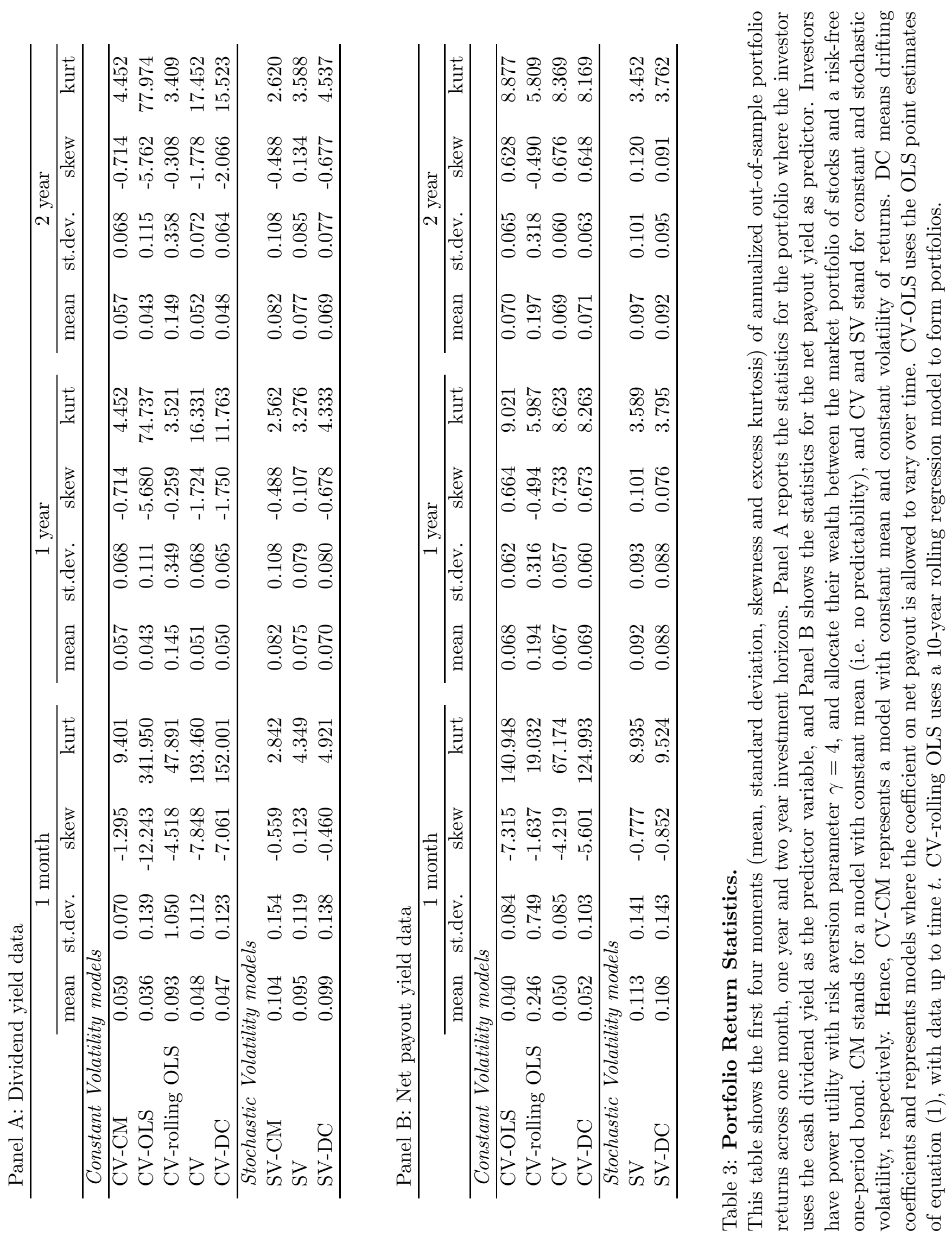
for volatility timing, assuming a constant mean. As mentioned earlier, there is no evidence in the literature for pure volatility timing over long time periods, although there is some evidence for a combination of volatility and correlation timing in the context of multiple asset portfolio problems. $^{14}$

Adding the dividend yield as a predictor, the full SV model does generate statistically significant improvements, where significance is at the $5 \%$ level and holds against both null models. This is our first evidence that an ensemble of factors improves performance, and also that there is an interaction between time-varying expected returns and time-varying volatility, as both are needed to generate statistically significant improvements. Compared to the CV-CM model that also incorporates parameter uncertainty, the CEs in the SV model are more than $1.5 \%$ higher and the monthly Sharpe ratios increase from 0.089 to 0.143 . Compared to the SV-CM model, the CEs increase by about $1 \%$ per year and the Sharpe ratios increase from 0.132 to 0.143 . The raw returns indicate that adding the dividend yield as a predictor improves skewness and decreases volatility, with only a minor reduction in average return and a mild increase in kurtosis.

At longer horizons, the returns generated by the SV model are often, but not always strongly statistically significant against both null models. The significance is slightly stronger for Sharpe ratios than for CEs for the longer horizon portfolios. The SV model has the least non-normalities, with slight positive skew and only modest excess kurtosis. Overall, we find strong evidence for statistically significant portfolio improvements at the short horizon using an ensemsble of factors and marginally significant evidence for the longer horizons.

The models with drifting coefficients perform extremely poorly with constant volatility and better with SV, but are not generally statistically significant against both null models, especially at longer investment horizons. This result should not be surprising, given the weak level of predictability and the additional parameters present in the DC models. We also note that both measures of model performance, the model probabilities and out-of-sample portfolios, identify the SV model as the best performing specification. Thus, the statistical and economic metrics coincide.

Some additional intuition for the relative performance can be seen from Figure 6, which shows the term structure of portfolio weights on a number of different dates, for the cumulative OLS model (CV-OLS) and the Bayesian models, using the dividend yield as predictor. The different models do generate very different long-horizon moments and return distributions, due to the timevarying state variables, estimation risk, and predictability. The differences arise because parameter uncertainty and mean-reversion (in expected returns and volatilities) impacts predictive moments differently as a function of investment horizon, as was discussed above.

The key difference between the cumulative OLS and our CV-CM benchmark model is the effect of parameter uncertainty. At short horizons the cumulative OLS and the CV weights are quite similar, but at longer horizons the CV weight tends to be below the cumulative OLS weight, reflecting the fact that parameter uncertainty effectively increases the volatility of the predictive

\footnotetext{
${ }^{14}$ See, e.g., Fleming, Kirby, and Ostdiek (2001, 2003). Their results are not fully out-of-sample, however, as they use in-sample information to estimate the presumed constant means. They also focus on a relatively short sample from 1983 to 1997.
} 

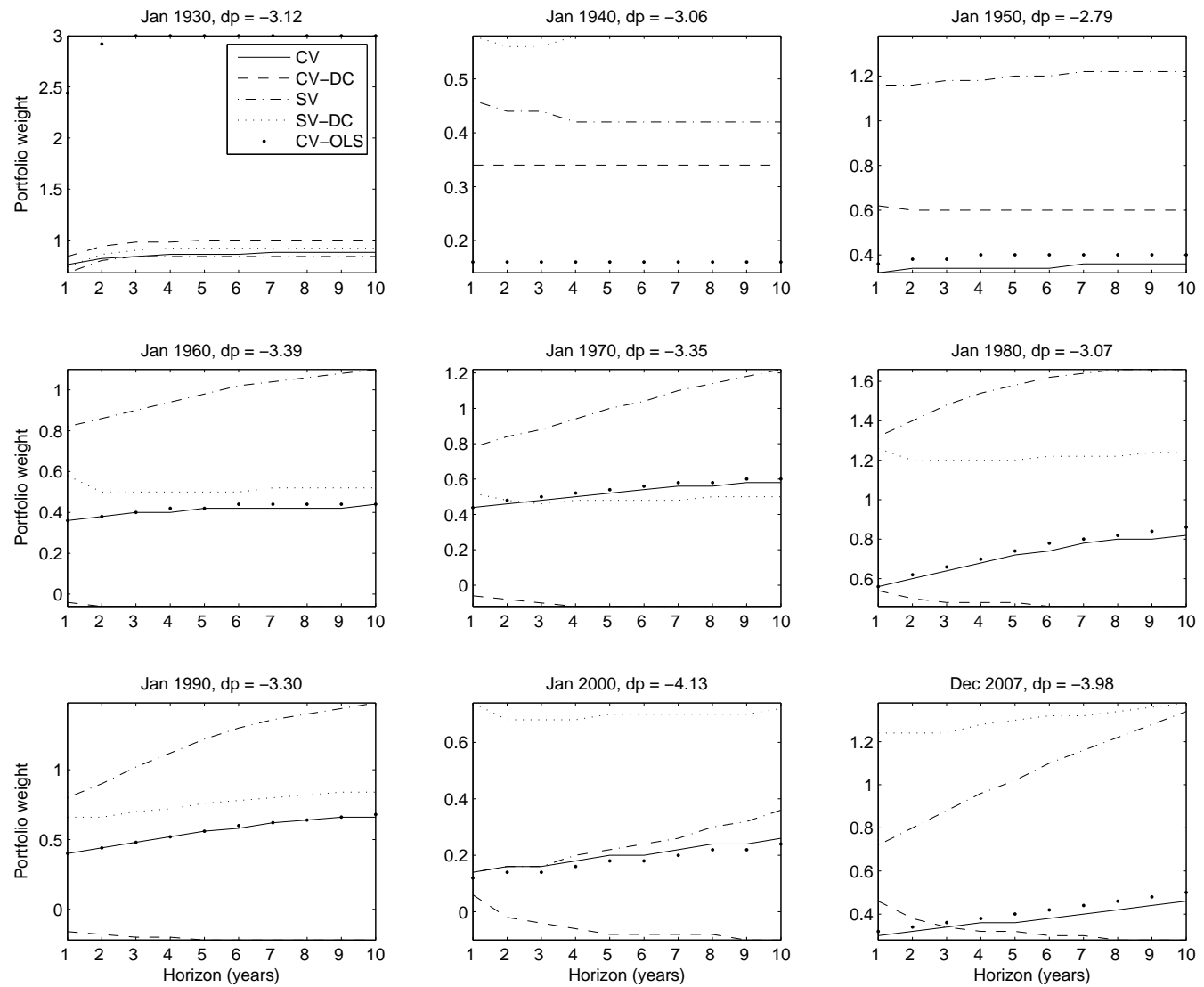

Figure 6: Optimal portfolio weights by investor horizon: Dividend yield data Plots of optimal portfolio weights for an investor who allocates wealth between the market portfolio of stocks and a risk-free one-period bond, with an investment horizon spanning from one to ten years. The plots shows the optimal weights on the stock portfolio at the beginning of each decade in our sample period, as well as at the final datapoint in our sample (December 2008, bottom-right plot). The investor has power utility with risk aversion $\gamma=4$, and rebalances annually while accounting for all parameter and state uncertainty. CV and SV represent models with expected return predictability and constant volatility (CV) and stochastic volatility (SV), respectively. DC stands for drifting coefficients and represents models where the predictability coefficient is allowed to vary over time. CV-OLS uses the OLS point estimates of equation (1), with data up to time $t$. 
market returns (as seen in Figure 4). The difference tends to decrease as time progresses and more information arrives, reducing the importance of parameter uncertainty. We also see that the models with stochastic volatility tend to have higher portfolio weights. This happens because the initial high variance of the 1930s is only slowly unlearned by the constant variance models, resulting in lower portfolio weights for much of the sample period. This underscores the importance of learning in a portfolio setting. The rolling regression portfolio weights are extremely variable and uninformative, with huge portfolio turnover, and for clarity we do not separately show them. The results for the net payout ratio are similar and are reported in the online appendix.

\subsubsection{Models using payout yields}

The portfolio results for the net payout predictor for each model are in Table 2 and Panel B of Table 3. The main result is that we find uniformly statistically significant evidence for performance improvement for the SV specification using net payout yields, as the out-of-sample CE yields and Sharpe ratios are significant for every risk aversion and horizon combination. The statistical significance is relative to both null models, which indicates that it is the combination of time-varying volatility and expected return predictability that generates the significance.

In terms of economic significance, the CE yields for the short horizon portfolios are $6.85 \%$ (5.71\%) for $\gamma=4(\gamma=6)$, respectively. This can be compared with a CE yield of $4.84 \%(4.43 \%)$ in the constant mean and variance case (from Table 1). Thus, expected return and volatility timing increases CE returns by $1.5 \%$ to $2 \%$ per year, which, when compounded over a long sample generates a dramatic, economically significant increase in realized utility. The monthly Sharpe ratios improve from 0.092 to roughly 0.155 , an improvement of nearly $70 \%$. The return statistics in Table 3 show strong improvement when compared to the CV specification at the one-month horizon, documenting the importance of time-varying volatility in controlling tail behavior. Thus, there is strong statistical and economic evidence for the ability to time the investment set when using an ensemble of factors.

For the constant volatility models, none of the CE yields are statistically significant against either benchmark model, and the Sharpe ratios are insignificant at the monthly horizon for both risk aversions. For short time-horizons, the bottom panel in Table 3 shows that these models generate low average returns and extremely negative skewness and very high kurtosis. Thus, at the one-month horizon, none of the constant volatility models generate any statistical significance.

At longer horizons, many of the constant volatility specifications are statistically significant when performance is measured by the Sharpe ratio, but always insignificant when measured by the CE yield. This curious result can be reconciled by the skewness and kurtosis statistics, both of which are ignored when computing Sharpe ratios. For example for the CV specification, the skewness is -4.2 and kurtosis is 70 . The CEs are insignificant for the constant volatility specifications because they take into account the higher moments due to the power utility specification. At longer horizons, the skewness improves for the constant volatility models, but the kurtosis is still generally greater than 10, which is heavily penalized in the CE metric, but not the Sharpe ratio metric. 
The drifting coefficients specification with time-varying volatility generates statistically significant gains in every risk-aversion and investment horizon case. Even though the drifting coefficients model has three additional parameters, the increased signal-to-noise ratio of the net payout measure combined with stochastic volatility is sufficient to accurately estimate the drifting coefficient. Although significant across both metrics and for all time-horizon/risk-aversion cases, the SV model performs better in every case. Thus, there is a small, statistically insignificant loss from adding a drifting coefficients specification.

Overall, the results indicate that statistically and economically significant gains are generated for every time horizon and risk aversion case provided that the investor (a) incorporates stochastic volatility, (b) incorporates expected return predictability using the net payout measure, and (c) accounts for parameter uncertainty when forming optimal portfolios. Together, this points toward the importance of an ensemble of factors that are required to generated statistically significant out-of-sample portfolio improvements from predictability models. Expected return timing alone, even when accounting for parameter uncertainty, does not generate significant gains.

\section{Conclusions}

This paper studies the problem of an investor who learns about the investment set over time with the goal of forming optimal portfolios, using various models that incorporate dividend yield based expected return predictors and stochastic volatility. The learning problem is Bayesian and is solved by using particle filters to generate samples from the posterior distribution of parameters, states, and models at each time period. After learning, our investor forms optimal portfolios by maximizing expected utility.

We reconcile seemingly contradictory evidence in the literature regarding the economic and statistical evidence for portfolio improvements generated by incorporating predictability. We find that an ensemble of factors that capture first-order important features of returns are needed to generate statistically significant portfolio improvements. In terms of models, it is important to incorporate stochastic volatility and time-varying expected returns, where the time-variation in expected returns is captured by the net payout ratio from Boudoukh et al. (2007). It is also important to account for parameter uncertainty when forming optimal portfolios. We corroborate the findings in Goyal and Welch (2008) that simple predictability models with constant volatility do not lead to statistically significant out-of-sample portfolio gains.

We also study the problem of sequential parameter inference and model monitoring, tracking relative model performance over time. We find strong time-variation in the investor's beliefs in parameters and over different model specifications. Moreover, these beliefs can change quite rapidly as new data arrives. These novel results highlight that learning about models, in practice, may not be a slow process, as outlying observations quickly revise beliefs. We also connect our results and models to the recent work by Pastor and Stambaugh (2009) on predictive systems and find that some of the specifications that we consider also have increasing volatility at longer horizons, even though the models are not necessarily predictive systems. These results suggest that the Pastor 
and Stambaugh result may be far more general than their specific predictive systems model.

There are a number of important directions in which the analysis can be extended. While we document statistically significant improvements in optimal portfolio performance, it should be possible to further improve the performance by allowing for multiple predictor variables, more general model specifications, and by incorporating economic restrictions as in Koijen and Van Binsbergen (2010). It would also be interesting to study optimal portfolios with alternative preferences that take into account a preference for early resolution of uncertainty, especially with model and parameter uncertainty. 


\section{References}

Andersen, T.G., L. Benzoni and J. Lund (2002). An Empirical Investigation of Continuous-Time Equity Return Models. Journal of Finance, 57, 1239-1284.

Ang, A. and G. Bekaert (2007). Stock Return Predictability: Is it There? Review of Financial Studies, 20, 651-707.

Avramov, D. (2002). Stock Return Predictability and Model Uncertainty. Journal of Financial Economics, 64, 423-458.

Bandi, F.M., J.R. Russell and Y. Zhu (2008). Using High-Frequency Data in Dynamic Portfolio Choice. Econometric Reviews, 27(1), 163-98.

Barberis, N. (2000). Investing for the Long Run when Returns are Predictable. Journal of Finance, $55,225-264$.

Boudoukh, J., R. Michaely, M. Roberts and M. Richardson (2007). On the Importance of Measuring Payout Yield: Implications for Empirical Asset Pricing. Journal of Finance, 62, 877-915.

Brandt, M.W., A. Goyal, P. Santa-Clara, J.R. Stroud (2005). A Simulation approach to Dynamic Portfolio Choice with an application to Learning about Predictability. Review of Financial Studies, $18,831-873$.

Brav, A., J.R. Graham, C.R. Harvey, and R. Michaely (2005). Payout Policy in the 21st Century. Journal of Financial Economics, 77, 483-527.

Brennan, M.J. (1998). The Role of Learning in Dynamic Portfolio Decisions. European Finance Review, 1, 295-306.

Campbell, J.Y., and R. Shiller (1988). Stock Prices, Earnings and Expected Dividends. Journal of Finance, 43, 661-676.

Campbell, J.Y., and S.B. Thompson (2008). Predicting Excess Stock Returns Out of Sample: Can Anything Beat the Historical Average. Review of Financial Studies, 21, 1509-1531.

Campbell, J.Y., and M. Yogo (2006). Efficient Tests of Stock Return Predictability. Journal of Financial Economics, 81, 27-60.

Carvalho, C., M.J. Johannes, H.F. Lopes, and N.G. Polson (2010). Particle Learning and Smoothing. Statistical Science, 25(1), 88-106.

Carvalho, C., M.J. Johannes, H.F. Lopes, and N.G. Polson (2011). Particle Learning for Sequential Bayesian Computation. Bayesian Statistics, 9, forthcoming. 
Cochrane, J. (2008). The Dog that did not Bark: A Defense of Return Predictability. Review of Financial Studies, 21, 1533-1575.

Cremers, M. (2002). Stock return Predictability: A Bayesian Model Selection Procedure. Review of Financial Studies, 15, 1223-49.

Dangl, T., and M. Halling (2011). Predictive Regressions with Time-Varying Coefficients. Journal of Financial Economics, forthcoming.

Das, S.R. and R.K. Sundaram (1999). Of Smiles and Smirks: A Term-Structure Perspective. Journal of Financial and Quantitative Analysis, 34, 211-239.

Eraker, B. Johannes, M., Polson, N.G. (2003). The Impact of Stochastic Volatility and Jumps in Returns. Journal of Finance, 1269-1300.

Ferson, W.E., S. Sarkissian and T. Simin (2003). Spurious Regressions in Financial Economics? Journal of Finance, 53, 1393-1413.

Fleming, J., C. Kirby and B. Ostdiek (1998). Information and Volatility Linkages in the Stock, Bond, and Money Markets. Journal of Financial Economics 49, 111-137.

Fleming, J., C. Kirby and B. Ostdiek (2001). The Economic Value of Volatility Timing. Journal of Finance 56, 329-352.

Fleming, Jeff, Chris Kirby and B. Ostdiek (2003). The Economic Value of Volatility Timing Using 'Realized' Volatility. Journal of Financial Economics 67, 473-509.

Goyal, A. and I. Welch (2008). A Comprehensive Look at the Empirical Performance of Equity Premium Prediction. Review of Financial Studies, 21, 1455-1508.

Hansen, L. (2007). Beliefs, Doubts and Learning: Valuing Macroeconomic Risk. American Economic Review. 97, 1-30.

Harvey, C.R. and A. Siddique (2000). Conditional Skewness in Asset Pricing Tests. Journal of Finance, 55, 1263-1295.

Harvey, C.R., J.C. Liechty, M.W. Liechty and P. Mueller (2010). Portfolio Selection with Higher Moments. Quantitative Finance, 10(5), 469-485.

Henkel, S.J., J.S. Martin and F. Nardari, Time-Varying Short-Horizon Return Predictability. Journal of Financial Economics, forthcoming.

Hodrick, R.J. (1992). Dividend Yields and Expected stock returns: alternative procedures for inference and measurement. Review of Financial Studies, 5, 357-386. 
Jacquier, E., N.G. Polson and P. Rossi (1994). Bayesian Analysis of Stochastic Volatility Models. Journal of Business and Economic Statistics, 12, 371-389.

Jacquier, E., N.G. Polson and P. Rossi (2005). Bayesian analysis of correlated stochastic volatility models. Journal of Econometrics, 122, 185-212.

Johannes, M. and Polson, N.G. (2006). Exact Particle Filtering and Parameter Learning. Working paper, University of Chicago.

Kandel, S. and R. Stambaugh (1996). On the predictability of stock returns: an asset allocation perspective. Journal of Finance, 51, 385-424.

Kim, S., N. Shephard and S. Chib (1998). Stochastic Volatility: Likelihood Inference and Comparison with ARCH Models. Review of Economic Studies, 65, 361-393.

Koijen, R. and J. Van Binsbergen, J (2010). Predictive Regressions: A Present-Value Approach. Journal of Finance, 65, 1439-1471.

Lettau, M., and S. Ludvigson (2001). Consumption, Aggregate Wealth, and Expected Stock Returns. Journal of Finance, 56, 815-850.

Lettau, M., and S. van Nieuwerburgh (2008). Reconciling the Return Predictability Evidence. Review of Financial Studies, 21(4), 1607-1652.

Lewellen, J. (2004). Predicting returns with financial ratios. Journal of Financial Economics, 74, 209-235.

Menzly, L., Santos, T., and P. Veronesi (2004). Understanding Predictability. Journal of Political Economy, 112, 1-47.

Pastor, L. and R. Stambaugh (2009). Predictive Systems: Living with Imperfect Predictors. Journal of Finance, 64, 1583-1628.

Pastor, L. and R. Stambaugh (2011), Are Stocks Really Less Volatile in the Long Run?, Journal of Finance, forthcoming.

Paye and Timmerman (2006). Instability of Return Prediction Models. Journal of Empirical Finance, 13, 274-315.

Rosenberg, B. (1972). The Behaviour of Random Variables with Nonstationary Variance and the Distribution of Security Prices. Working paper, University of California, Berkeley.

Santos, T. and P. Veronesi (2006). Labor Income and Predictable Stock Returns. Review of Financial Studies, 19, 1-44. 
Shanken, J.A. and A. Tamayo (2011). Dividend yield, Risk and Mispricing: a Bayesian analysis. Journal of Financial Economics, forthcoming.

Shiller, R.J. (1981). Do stock prices move too much to be justified by subsequent changes in dividends? American Economic Review, 71, 421-436.

Stambaugh, R. (1986). Bias in regressions with lagged stochastic regressors, CRSP Working paper, 156, University of Chicago.

Stambaugh, R. (1999). Predictive Regressions. Journal of Financial Economics, 54, 375-421.

Torous, W., R. Valkanov, and S. Yan (2004). On Predicting Stock Returns with Nearly Integrated Explanatory Variables. Journal of Business, 77, 937-966.

Wachter, J.A. and M. Warusawitharana (2009). Predictable returns and asset allocation: should a skeptical investor time the market? Journal of Econometrics, 148(2), 162-178.

Wachter, J.A. and M. Warusawitharana (2011). What is the Chance that the Equity Premium Varies over Ttime? Evidence from Predictive Regressions. Working paper, University of Pennsylvania Wharton School of Business.

Xia, Y. (2001). Learning about Predictability: the effect of parameter learning on dynamic asset allocation. Journal of Finance, 56, 205-246.

Yan, (2005). Asymmetry, Fat-tail, and Autoregressive Conditional Density in Financial Return Data with Systems of Frequency Curves. Working paper, University of Iowa. 